\title{
5. CRETACEOUS PLANKTONIC FORAMINIFERS AND DEPOSITIONAL ENVIRONMENTS FROM THE ONTONG JAVA PLATEAU WITH EMPHASIS ON SITES 803 AND $807^{1}$
}

\author{
William V. Sliter ${ }^{2}$ and R. Mark Leckie ${ }^{3}$
}

\begin{abstract}
Cretaceous planktonic foraminifers from Ocean Drilling Program (ODP) Leg 130 consist of two age groups: one dominantly Aptian in age and the other dominantly Maastrichtian. The oldest dated sediments from Ontong Java Plateau occur at ODP Site 807 and are assigned to the early Aptian Globigerinelloides blowi Zone. The assemblage correlates closely with Aptian foraminifers from Deep Sea Drilling Project (DSDP) Site 289 and a younger Aptian assemblage from DSDP Site 288 where drilling ended before reaching basement. Planktonic foraminifers from ODP Site 803 suggest a late Aptian to early Albian age and are overlain by radiolarians of late Albian age. These ages are in conflict with a radiometric age of about $90 \mathrm{Ma}$ for basement at the site. The dominantly Maastrichtian group occurs at Site 807 and consists of poorly preserved, mostly small-sized specimens that range in age from perhaps the latest Campanian to the Cretaceous/Tertiary boundary. Age assignments are made difficult by pervasive dissolution and resedimentation that extends into the Paleogene.

Deposition of the Aptian limestone at Site 807 took place at lower bathyal depths beneath the foraminiferal lysocline but above the calcite compensation depth (CCD). Deposition then continued beneath the CCD until the late Campanian or Maastrichtian when carbonate sedimentation resumed. Within the Maastrichtian, deposition again took place at lower bathyal depths at or above the rapidly dropping $\mathrm{CCD}$ until the Cretaceous/Tertiary boundary when the $\mathrm{CCD}$ apparently rose. Small planktonic foraminifers accumulated in winnowed and redeposited fine-grained sediments whereas larger planktonic foraminifers were deposited largely in mass-flow deposits that increase in number upsection through the Maastrichtian. The increased pulses of larger planktonic foraminifers followed by the abrupt occurrence of laminated sediments, an abundance of small, globular planktonic foraminifers, and an increase in radiolarians in the latest Maastrichtian likely were the result of northward plate motion into more productive waters and different current systems. At Site 803, deposition beneath the CCD continued from the earliest sediments above the basalt until the Eocene. Carbonate sedimentation during the late Albian to early Santonian, however, is recorded atop the plateau at Site 288

The extensive hiatuses in the Cretaceous sequence from the Ontong Java Plateau correlate in part with times of widespread hiatuses in the Pacific Basin. Evidence of pervasive mechanical erosion during the Cenomanian to Santonian and the Campanian to Maastrichtian on the top of the plateau perhaps were caused by the longitudinal position of the plateau relative to western boundary currents and eddy effects above the plateau.
\end{abstract}

\section{INTRODUCTION}

Cretaceous planktonic foraminifers were recovered from Sites 803 and 807 drilled on the northern part of the Ontong Java Plateau (Fig. 1) during Ocean Drilling Program (ODP) Leg 130. These and similar planktonic foraminifers described from Sites 288 and 289 of Deep Sea Drilling Project (DSDP) Leg 30 (Sliter, 1992) occur in the sedimentary sequence that directly overlies basement and thus provide a minimum age for the cessation of volcanism and a means to interpret the initial depositional environment.

The timing of volcanism that formed the immense Ontong Java Plateau $\left(>1.5 \times 10^{6} \mathrm{~km}^{2}\right)$ recently has come under scrutiny as it pertains to both the plume initiation model of large oceanic basalt plateaus (Richards et al., 1991) and the eruptive rate of the oceanic flood basalt events that apparently occurred within a few million years (Tarduno et al., 1991; Whitechurch et al., 1992). In addition, the massive and sudden outpouring of submarine basalts during the emplacement of large oceanic plateaus may be linked to global changes in sea level and climate (Caldeira and Rampino, 1990, 1991; Arthur et al., 1990; Larson, 1991a, 1991b; Tarduno et al., 1991). Thus, establishing the temporal record of volcanism at Ontong Java Plateau, the world's largest oceanic plateau, offers perhaps the best means to

\footnotetext{
${ }^{1}$ Berger, W.H., Kroenke, L.W., Mayer, L.A., et al., 1993. Proc. ODP, Sci. Results, 130: College Station, TX (Ocean Drilling Program).

${ }^{2}$ U.S. Geological Survey, 345 Middlefield Road MS 915, Menlo Park, CA 94025, U.S.A.

${ }^{3}$ Department of Geology and Geography, University of Massachusetts, Morrill Science Center, Amherst, MA 01003, U.S.A.
}

investigate the environmental consequences of a major submarine volcanic event.

The Cretaceous foraminifers and associated biogenic material also provide a means to assess the initial depositional environment on the Ontong Java Plateau. Two aspects are of particular interest. First, an estimation of the water depth of the earliest sediments following the cessation of volcanism is needed. This is important in determining the pre- and post-emplacement vertical motion history of the plateau. Second, and equally important, the sediments record a rare glimpse of southern mid-latitude paleoceanographic conditions in the Cretaceous Pacific Ocean as the plateau moved northward with the Pacific Plate from a paleolatitude of about $40^{\circ} \mathrm{S}$ in the Aptian, to about $17^{\circ} \mathrm{S}$ in the Maastrichtian (Gordon, 1990).

Here we present the Cretaceous planktonic foraminiferal biostratigraphy and environmental interpretation for Leg 130. Results from Sites 803 and 807 are compared with those from a reexamination of samples from DSDP Sites 288 and 289. Primary emphasis is placed on the oldest samples from the four sites and the younger section from Site 807. Stratigraphic distributions of Cretaceous foraminifers in Hole 807C are shown in Tables 1 and 2, selected species are illustrated in Plates $1-4$, and a faunal reference list is given in the Appendix.

\section{METHODS}

A total of 91 samples from Leg 130 were examined in thin section in addition to a few isolated specimens that were derived from crushed limestone or washed samples. In addition, seven samples from upper Aptian limestone of Site 289 were crushed and examined for whole specimens of planktonic foraminifers. Samples for thin sections were selected randomly throughout most of the cored intervals. Most of the 


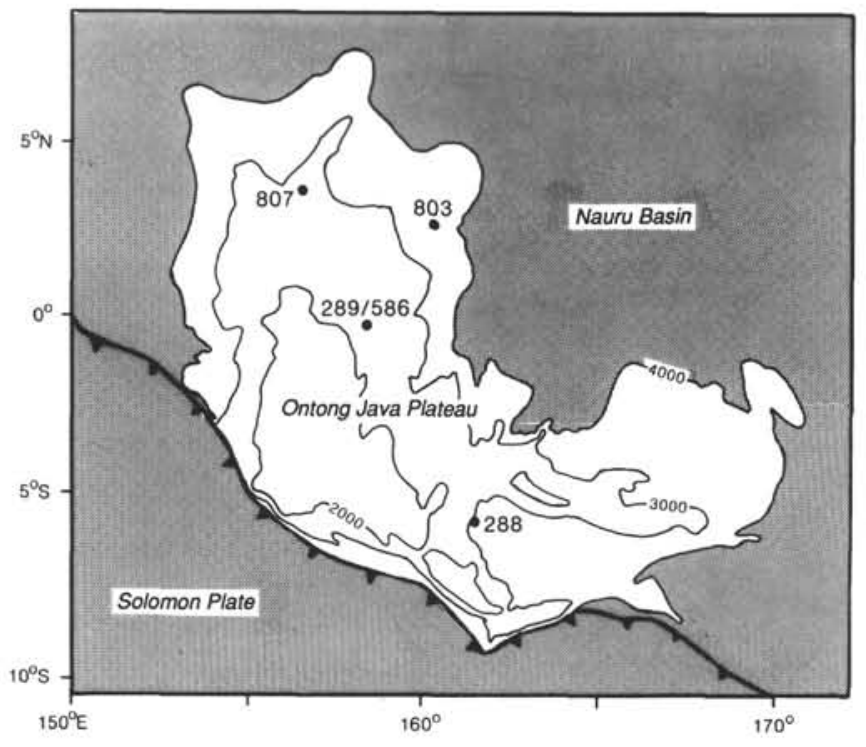

Figure 1. Location of drilling sites on Ontong Java Plateau that contain Cretaceous planktonic foraminifers.

sections were cut parallel to the core whereas a few were cut parallel to bedding and are so indicated on the following tables because the orientation of the sections affects the character (i.e., species composition, diversity, specimen size, etc.) of the foraminiferal assemblage in nonhomogeneous sediments. The stratigraphic distribution of planktonic foraminifers in Tables 1 and 2 is shown by their presence or absence. For thin section examination, the relative abundance of planktonic and benthic foraminifers and radiolarians was estimated by scanning the section at a magnification of about $150 \times$ using the following scale:

$$
\begin{aligned}
& A=\text { abundant }(>20 \% \text { of the total assemblage }), \\
& C=\text { common }(10 \%), \\
& F=\text { few }(5 \%), \\
& R=\text { rare }(1 \%), \text { and } \\
& \text { VR = very rare }(<1 \% \text { or } 1-3 \text { specimens })
\end{aligned}
$$

Preservation was determined qualitatively based on the degree of dissolution and/or overgrowth exhibited by the planktonic foraminifer and radiolarian assemblages examined in thin section. The following scale was used for planktonic foraminifers:

$\mathrm{G}=$ good (individual specimens exhibit little dissolution or recrystallization);

$\mathrm{M}=$ moderate (dissolution and recrystallization [overgrowth] of some individuals is apparent; species identifications are usually not impaired);

$\mathrm{P}=$ poor (specimens exhibit strong dissolution and/or recrystallization; many specimens are represented by relicts or "ghosts" that make identifications impossible); and

$\mathrm{VP}=$ very poor (most specimens are represented by ghosts; species identifications are rare to very rare; generic identifications may be rare).

Sample notation follows the standard ODP format given as drilling leg followed by site, core, core type ( $R=$ rotary), section, and interval (centimeters below top of section). The abbreviation "CC" refers to a core-catcher sample taken from the bottom of the cored interval.

\section{BIOCHRONOLOGY AND GEOCHRONOLOGY}

The biozonation used is modified from that of Sliter (1989) for

\begin{tabular}{|c|c|c|c|c|c|c|c|c|c|c|c|c|}
\hline $\begin{array}{l}\text { Core, section, } \\
\text { interval }(\mathrm{cm})\end{array}$ & 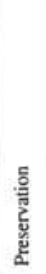 & 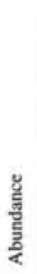 & $\begin{array}{l}\frac{\sqrt{6}}{\frac{5}{8}} \\
\frac{0}{5}\end{array}$ & 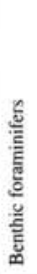 & 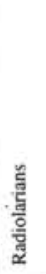 & 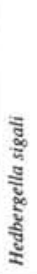 & 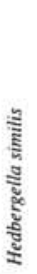 & 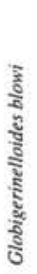 & 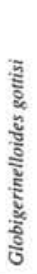 & 竎 & 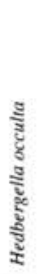 & 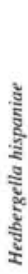 \\
\hline \multicolumn{13}{|l|}{ 130-803D- } \\
\hline $72 R-1,47-99$ & VP & . & 100 & . & F & s & . & . & . & . & . & . \\
\hline $72 R-1.72-74$ & VP & . & 90 & & C & . & . & . & . & . & . & . \\
\hline $72 R-2,30-32$ & VP & . & 85 & & $\mathrm{~F} / \mathrm{C}$ & & & , & . & . & . & . \\
\hline $72 R-3,11-13$ & VP & & 85 & . & C & . & & & . & $\cdot$ & & . \\
\hline 72R-CC & & . & . & . & $\cdot$ & . & & . & . & . & - & . \\
\hline $73 \mathrm{R}-1,55-57$ & VP & . & 90 & . & F/C & . & & & . & . & . & . \\
\hline $73 R-1,113-i 15$ & VP & . & 90 & $\mathbf{R}$ & C & i. & . & . & . & . & . & . \\
\hline $73 R-2,103-105$ & VP & $\mathbf{R}$ & 80 & $\mathbf{R}$ & & $\mathrm{x}$ & $\mathrm{x}$ & & & & . & . \\
\hline $73 R-2,115-117$ & VP & $\mathbf{R}$ & 70 & $\mathbf{R}$ & . & $\mathrm{x}$ & & $\mathrm{x}$ & . & . & . & $\mathrm{x}$ \\
\hline 73R-CC & VP & $\mathbf{R}$ & 70 & $\mathbf{R}$ & . & $\mathrm{x}$ & $\mathrm{x}$ & . & . & . & . & $\mathrm{x}$ \\
\hline $74 \mathrm{R}-1,23-25$ & VP & $\mathbf{R}$ & 40 & $\mathbf{R}$ & VR & $\mathrm{x}$ & $\mathrm{X}$ & $\mathrm{x}$ & $\mathrm{X}$ & cf & $\mathrm{cf}$ & . \\
\hline$* 80 R-1,78-80$ & VP & . & 100 & . & C & . & 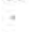 & . & . & , & . & - \\
\hline $80 R-1,93-95$ & VP & . & 100 & & $\mathrm{~F} / \mathrm{C}$ & . & . &. & . & . & . & . \\
\hline $80 R-1,99-101$ & VP & & 100 & . & VR & . & & . & . & . & . & . \\
\hline $80 \mathrm{R}-1,111-114$ & VP & . & 100 & . & C & . & . & & . & - & & \\
\hline
\end{tabular}
Cretaceous planktonic foraminifers examined in thin section (Fig. 2). Species identifications in thin section are based on an evolutionary
Table 1. Occurrence of Early Cretaceous foraminifers and radiolarians, Hole 807C.

Notes: Preservation: $V P=$ very poor. Abundance: $V R=$ very rare, $R=$ rare, $F=$ few, and $C=$ common. of $=$ probably identification. $X=$ present. An asterisk $(*)$ indicates thin section cut horizontal to bedding.

sequence of morphologic criteria recognizable in axial (parallel to the axis of coiling) or transverse (perpendicular to the axis of coiling) sections. These include test size and shape, the character of the test wall and periphery, chamber shape, number and arrangement, and the forms of ornamentation. The result is a zonal scheme based on the stratigraphic distribution of planktonic foraminifers that is correlated to the magneto-geochronology of Harland et al. (1982) and Kent and Gradstein (1985).

Of particular interest to this study is the age of the lower Aptian samples, and thus the age of Reversed-Polarity Chron M0 and the Barremian/Aptian boundary (Fig. 2). The ages of the early Aptian and Barremian events, however, remain speculative. Previously, in terms of microfossils, the Barremian/Aptian boundary was defined by the first appearance of the planktonic foraminifer Hedbergella similis and the calcareous nannofossils Rucinolithus irregularis and Chiastozygus litterarius following the zonations of Sigal (1977) and Thierstein (1973), respectively. The Hedbergella similis Zone was followed by the first occurrence (FO) of the planktonic foraminifer genus Globigerinelloides in the $G$. duboisi Zone and $G$. blowi in the succeeding G. blowi Zone, as shown in Figure 2. Reversed-Polarity Chron M0 was placed in the early Aptian Hedbergella similis Zone following the study of Channell et al. (1979) and calibrated to $118.2 \mathrm{Ma}$ according to Harland et al. (1982), who further dated the Barremian/Aptian boundary at $119 \mathrm{Ma}$.

Recent information has cast doubt on these placements. The revised time scale of Harland et al. (1990) now places the Hedbergella similis Zone in the latest Barremian and dates the top of ReversedPolarity Chron M0 at $124 \mathrm{Ma}$ and the Barremian/Aptian boundary, still defined by the first appearance of Chiastozygus litterarius, at 124.5 Ma. Little information is given as to the reasons for revising the microfossil biostratigraphy (see discussion in Tarduno et al., 1991). In fact, revision of the Barremian to early Aptian planktonic foraminiferal datums is required based on new data from Leckie and Bralower (1991) and Coccioni et al. (1992). Leckie and Bralower (1991), in a study of Atlantic DSDP and ODP sites, indicate that the FO of Globigerinelloides is in the early Barremian, whereas the FO of Rucinolithus irregularis still marks the Barremian/Aptian boundary and the FO of Globigerinelloides blowi remains in the early Aptian. Furthermore, the FO of Rucinolithus irregularis occurs be- 
fore Reversed-Polarity Chron M0, as was demonstrated for Pacific DSDP sites by Tarduno et al. (1989). In contrast, Coccioni et al. (1992) correlate the Hedbergella similis Zone with Chron M2, the Globigerinelloides duboisi Zone with Chron M1, and place the FO of G. blowi in the late Barremian before the FO of Chiastozygus litterarius that continues to mark the Barremian/Aptian boundary based on a study of the Gorgo Cerbara section in Italy. Importantly, their study shows that a major part of the $G$. blowi Zone remains in the Aptian and continues to include Chron M0. Obviously, further study is needed to resolve these ambiguities.

The continued placement of Reversed-Polarity Chron M0 and at least a major part of the Globigerinelloides blowi Zone in the early Aptian is important to this study as our oldest samples are assigned to the G. blowi Zone. Paleomagnetic data from Site 807 show that the basalt and overlying limestone are of normal polarity (Tarduno et al., 1991; Mayer and Tarduno, this volume) and, as dated paleontologically, likely magnetized early in the long Cretaceous Normal Polarity Superchron. Further, ${ }^{40} \mathrm{Ar} /{ }^{39} \mathrm{Ar}$ ages from basalt at Sites 289 and 807 yield ages that are indistinguishable around a mean of $122 \mathrm{Ma}$ (Mahoney et al., this volume). Thus, although the geochronologic ages are similar to those of Harland et al. (1990), we lack the means at present to correlate the paleontologic datums during the Barremian and early Aptian. Accordingly, in this paper we continue to use the correlations of Harland et al. (1982) and Kent and Gradstein (1985).

\section{BIOSTRATIGRAPHY}

The biostratigraphy presented here represents the post-cruise analysis of Holes $803 \mathrm{D}$ and $807 \mathrm{C}$ and is summarized in Figure 3. Cretaceous limestone samples with planktonic foraminifers from both holes are divided into two age groups: a dominantly Aptian group and a Late Cretaceous group. These two groups are separated by an interval of red and brown claystone and siltstone that yields radiolarian assemblages of early Aptian and late Albian age at Sites 807 and 803 , respectively (Takahashi and Ling, this volume). Similarly, at DSDP Sites 288 and 289 (Fig. 3), Aptian and Late Cretaceous assemblages of planktonic foraminifers and calcareous nannofossils are separated by major hiatuses; however, at Hole 288A the intervening mid-Cretaceous interval contains planktonic foraminifers (Sliter, 1992). Thus, in this and the following section on depositional environments, we based our discussion on the age groupings shown in Figure 3. Furthermore, we examined the faunal assemblages separately in terms of their age and environment although we acknowledge that the environment on Ontong Java Plateau has exerted an unusually strong influence on the recovery and composition of the faunas throughout its history of deposition.

\section{Age and Correlation of the Oldest Sediments}

\section{Hole $803 \mathrm{D}$}

Hole 803D, at a water depth of $3412.2 \mathrm{~m}$, is one of four holes drilled at Site 803 located about $400 \mathrm{~km}$ northeast of DSDP Site 289 on the northeastern margin of the Ontong Java Plateau (Fig. 1). Seventy-one cores were taken, with Cretaceous sediment and basaltic basement recovered in Core 130-803D-68R. The top of the basalt is $4043.2 \mathrm{~m}$ below the present sea level. The basalt is capped by a 2-cm-thick, layered deposit of questionable hydrothermal origin (Kroenke, Berger, Janecek, et al., 1991) followed by dark brown claystone with laminae and several thin layers of radiolarite in Section 130-803D-68R-3 that grade upward into homogeneous claystone (Fig. 4). The presence of several thin interpillow limestone fragments that contain recrystallized microfossils (see Hole $807 \mathrm{C}$ below) may indicate a hiatus at the basalt/sediment contact caused by the erosion of the initial carbonate sediments.

Cretaceous planktonic foraminifers from this hole are limited to rare specimens of Hedbergella sigali in an assemblage of mostly agglutinated foraminifers washed from Sample 130-803D-68R-3, $143-145 \mathrm{~cm}$. Hedbergella sigali ranges in age from the Barremian to the early Albian and is most abundant in the Aptian. An Aptian to early Albian age for the foraminifers is suggested by both the occurrence of late Albian radiolarians immediately above in Sample 130-803D68R-3, 89-90 cm (Takahashi and Ling, this volume), as well as by the similarity in lithology of the claystone unit at Site 803 to the upper part of the corresponding claystone-siltstone unit at Site 807 (Fig. 4).

The paleontologic age of this interval, however, is problematic in light of the ${ }^{40} \mathrm{Ar} /{ }^{39} \mathrm{Ar}$ date of about $90 \mathrm{Ma}$ for basement at Site 803 (Mahoney et al., this volume). Possible reworking of the microfossils by mass transport events might explain the age discrepancy in this condensed section. This explanation, however, seems unlikely as it requires that both the foraminifers and radiolarians, which occur in separate samples, were reworked in stratigraphic sequence, the opposite of what might be expected (Fig. 3). The condensed nature of the Cretaceous claystone interval at Site 803 is verified by the position of the Cretaceous/Tertiary boundary located near Sample 130-803D$68 \mathrm{R}-1,46.5 \mathrm{~cm}$, based on calcareous nannofossils (Kroenke, Berger, Janecek, et al., 1991).

\section{Hole $807 C$}

Hole $807 \mathrm{C}$ is one of three holes drilled at Site 807 on the northern rim of the high plateau (Fig. 1). It is located at a water depth of $2805.7 \mathrm{~m}$ within a shallow basement graben about $0.5 \mathrm{~km}$ from the foot wall on the northern side of the graben. Ninety-three cores were recovered with basalt encountered in Core $130-807 \mathrm{C}-74 \mathrm{R}$ at $1380 \mathrm{~m}$ below seafloor $(4185.4 \mathrm{~m}$ below present sea level). The basalts are tholeiites and consist mainly of pillow lavas and thin massive flows with one $28-\mathrm{m}$ massive flow (Kroenke, Berger, Janecek, et al., 1991). Two thin limestone interbeds were recovered in Cores 130-807C-82R and -80R. Examination of limestone samples from Core $130-807 \mathrm{C}-80 \mathrm{R}$ yielded common but very poorly preserved and recrystallized radiolarians (Table 1).

The stratigraphic sequence overlying the basalt in Cores 130$807 \mathrm{C}-74 \mathrm{R}$ to $-71 \mathrm{R}$ consists of moderately bioturbated limestone that grades upward into claystone and siltstone, as shown in Figure 4. Planktonic foraminifers are limited to the limestone that occurs in the top of Section 130-807C-74R-1 and the bottom of Section 130-807C73R-2. As shown in Figure 4, the exact meter level of the lowest limestone above the basalt is unknown, and the sample could have come from anywhere in the interval between the bottom of Core 130-807C-73R and the top of the basalt. The lithology of the limestone in the two cores differs, however, with that of Core 130-807C$74 \mathrm{R}$, which is nearly homogeneous with a single, light brown lamina and some mottling, whereas that of Core 130-807C-73R is distinctly laminated with red-brown claystone that increases in abundance upsection. The assemblage from the lowermost limestone, in Sample $130-807 \mathrm{C}-74 \mathrm{R}-1,23-25 \mathrm{~cm}$, is assigned to the early Aptian Globigerinelloides blowi Zone by the presence of the nominal species, G. gottisi and Hedbergella similis among others, and the probable occurrence of $H$. occulta (Table 1). The small size of the specimens and the low species diversity supports the zonal assignment (Sliter, 1989). Samples from Section 130-807C-73R-CC and -73R-2 again are dominated by small specimens of Hedbergella with the addition of rare medium-sized specimens attributed to $H$. hispaniae. The presence of these forms suggests an age assignment to the overlying Leupoldina cabri Zone. The difference in age and lithology clearly distinguishes the limestone resting on the basalt from that of Core 130-807C-73R. The Aptian age of Section 130-807C-73R-CC is confirmed by the presence of the calcareous nannofossil Rucinolithus irregularis that first occurs at the Barremian/Aptian boundary (Kroenke, Berger, Janecek, et al., 1991).

The limestone in Section 130-807C-73R-2 grades upward into claystone followed by an interval of claystone and interbedded radiolarian siltstone that extends from Sections 130-807C-73R-1 to -71R-6 and in turn is followed by more homogeneous claystone. Samples 
Table 2. Occurrence of Late Cretaceous foraminifers and radiolarians, Hole 807C.

\begin{tabular}{|c|c|c|c|c|c|c|c|c|c|c|c|c|c|c|c|c|c|c|c|c|c|c|}
\hline \multirow[b]{3}{*}{$\begin{array}{l}\text { Core, section, } \\
\text { interval (cm) }\end{array}$} & \multirow[b]{3}{*}{ 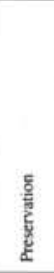 } & \multicolumn{5}{|c|}{ Foraminifers } & \multirow[b]{3}{*}{ 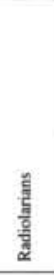 } & & & & & & & & & & & & & $\frac{\pi}{\xi}$ & & \\
\hline & & & nktonic & & & & & & & & & & & & & & & 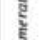 & & है้ & & \\
\hline & & 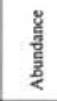 & 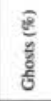 & $\begin{array}{l}\frac{\mathrm{y}}{\mathrm{o}} \\
\frac{\mathrm{g}}{0}\end{array}$ & 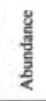 & $\begin{array}{l}\text { हूँ } \\
\text { है } \\
\text { है }\end{array}$ & & 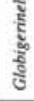 & 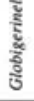 & t) & 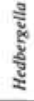 & $\begin{array}{l}\text { हैँ } \\
\text { हैँ } \\
\text { है }\end{array}$ & 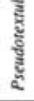 & $\begin{array}{l}\text { है. } \\
\text { है } \\
\text { हैं }\end{array}$ & 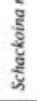 & 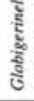 & हैँ & 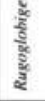 & हू & 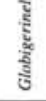 & $\frac{\mathrm{s}}{\mathrm{s}}$ & ถู้ \\
\hline 130-807- & & & & & & & & & & & & & & & & & & & & & & \\
\hline$\cdot 55 \mathrm{R}-1,55-50$ & vp & C/A & 95 & & VR & & & $\mathrm{x}$ & $\mathrm{x}$ & & & & $\mathrm{x}$ & & & & of & of & $\mathrm{x}$ & & & \\
\hline $55 R-1,65-66$ & $\mathrm{vP}$ & $\mathrm{F}$ & 95 & & VR & & VR & $x$ & $\mathrm{x}$ & $x$ & $x$ & $\hat{x}$ & $\hat{x}$ & & & & & & & $\hat{x}$ & & . \\
\hline$=55 R-1,97-98$ & vp & $\mathrm{F}$ & 100 & & & & & $x$ & $x_{x}^{x}$ & $x$ & $\begin{array}{l}x \\
x \\
x\end{array}$ & 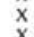 & $x$ & & i & $x$ & : & $\dot{x}$ & & $\dot{x}$ & & . \\
\hline $\begin{array}{l}\text { SSR-1, 103-105 } \\
-55 R-2,12-13\end{array}$ & $\begin{array}{l}\text { VP } \\
\text { vP }\end{array}$ & & $\begin{array}{r}100 \\
85\end{array}$ & & $\begin{array}{l}\text { VR } \\
\text { VR }\end{array}$ & $\mathrm{x}$ & $F$ & $\begin{array}{l}x \\
x\end{array}$ & $\begin{array}{l}x \\
x\end{array}$ & $\begin{array}{l}x \\
x\end{array}$ & $\begin{array}{l}x \\
x\end{array}$ & $\begin{array}{l}x \\
x\end{array}$ & $\begin{array}{l}x \\
x\end{array}$ & & & $\mathrm{x}$ & & & & $\begin{array}{l}x \\
x\end{array}$ & & \\
\hline 55R-2. 93-95 & vP & A & 90 & & & & & $\mathrm{x}$ & $\mathrm{x}$ & $\mathrm{x}$ & $\mathrm{x}$ & $\mathrm{x}$ & $\mathrm{x}$ & & & & & $\mathrm{x}$ & & $\mathrm{x}$ & & $\mathrm{x}$ \\
\hline $\begin{array}{l}\text { SSR }-3,74-76 \\
\text { SSR }-4,75-77\end{array}$ & vP & FiC & 95 & D & & & $R_{R}$ & $x$ & $\begin{array}{l}x \\
x\end{array}$ & $x_{x}^{x}$ & $x$ & $\begin{array}{l}x \\
x \\
x\end{array}$ & $x_{x}$ & & & $x$ & $\mathrm{x}$ & of & : & $\mathrm{x}$ & & 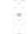 \\
\hline $\begin{array}{l}55 R-4,75-77 \\
\text { S6R-1, 10-12 }\end{array}$ & $\begin{array}{l}\mathrm{VP} \\
\mathrm{VP}\end{array}$ & ${ }_{\mathrm{A}}^{\mathrm{F}}$ & $\begin{array}{l}100 \\
90\end{array}$ & D & VR & & $R$ & $\begin{array}{l}x \\
x\end{array}$ & $\begin{array}{l}x \\
x\end{array}$ & $\begin{array}{l}x \\
x\end{array}$ & $\begin{array}{l}x \\
x\end{array}$ & $\begin{array}{l}x \\
x\end{array}$ & $\begin{array}{l}x \\
x\end{array}$ & $\mathrm{x}$ & & $\mathrm{x}$ & & $\mathrm{x}$ & & $x$ & $x$ & \\
\hline $56 \mathrm{R}-2,13-15$ & VP & & 95 & & & & $R / F$ & $x$ & $\hat{x}$ & $\hat{x}$ & $\hat{x}$ & $\hat{x}$ & $\hat{x}$ & & & & & of & & $\hat{x}$ & & $\mathrm{x}$ \\
\hline S6R-3.13-15 & vp & A & 90 & & VR & & & $x$ & $x$ & $\mathrm{x}$ & $\mathrm{x}$ & $\mathrm{x}$ & $\mathrm{x}$ & & & $\mathrm{x}$ & & $\mathrm{x}$ & & $x$ & & . \\
\hline $\begin{array}{l}57 R-C C \\
\text { SRPA }\end{array}$ & $\stackrel{p}{p}$ & A & 80 & & VR & $\mathrm{x}$ & & $x$ & $\mathrm{x}$ & $x$ & $x$ & $x$ & $\mathrm{x}$ & $x$ & & $\mathrm{x}$ & $x$ & $x$ & & $\mathrm{x}$ & & \\
\hline $\begin{array}{l}588 \cdot 1,33-35 \\
58 \cdot C C\end{array}$ & VP/P & c & 85 & & VR & & & $x$ & $x$ & $x_{x}^{x}$ & $x_{x}^{x}$ & $x_{x}^{x}$ & $\mathrm{x}$ & $\mathrm{x}$ & & $\mathrm{x}$ & $x_{x}^{x}$ & $\mathrm{x}$ & & $\dot{x}$ & $\mathrm{x}$ & \\
\hline S9R-1. 77-78 & VPP/P & c & 90 & & & & & $\mathrm{x}$ & $\hat{x}$ & $\hat{x}$ & $\hat{x}$ & $\hat{x}$ & $\mathrm{x}$ & $\mathrm{x}$ & & & $\begin{array}{l}x \\
x\end{array}$ & & & $\hat{x}$ & $\mathrm{x}$ & $\mathrm{x}$ \\
\hline $59 \mathrm{R}-2.99-100$ & VP/P & $\mathbf{F}$ & 90 & & VR & & & $\mathrm{x}$ & $\mathrm{x}$ & $\mathrm{x}$ & $\mathrm{x}$ & $\mathrm{x}$ & $\mathrm{x}$ & & & $x$ & $\mathrm{x}$ & of & & $x$ & $x$ & \\
\hline S9R-CC & $\mathrm{p}$ & c & 90 & . & $\mathrm{VR}$ & $\mathrm{x}$ & & $x$ & $\ddot{x}$ & $\hat{x}$ & $\hat{x}$ & $\hat{x}$ & $\hat{x}$ & $\mathrm{x}$ & & $\hat{x}$ & $\dot{x}$ & $x$ & & $\hat{x}$ & $\hat{x}$ & \\
\hline $60 R-1.4-5$ & vp & FIC & 90 & & $\begin{array}{l}\text { VR } \\
\text { VR }\end{array}$ & & & $x$ & $\mathrm{x}$ & $x$ & $x$ & $x$ & $\mathrm{x}$ & $x$ & & i & $\mathrm{x}$ & $\therefore$ & & & $x$ & $x$ \\
\hline $\begin{array}{l}\text { 60R-CC } \\
61 R-1.50-52\end{array}$ & & & $\begin{array}{l}90 \\
80\end{array}$ & & & $\mathrm{x}$ & & $\begin{array}{l}x \\
x\end{array}$ & $\begin{array}{l}x \\
x\end{array}$ & $\begin{array}{l}x \\
x\end{array}$ & $\begin{array}{l}x \\
x\end{array}$ & $\begin{array}{l}x \\
x\end{array}$ & $x$ & $\begin{array}{l}x \\
x\end{array}$ & & $\begin{array}{l}x \\
x\end{array}$ & & $\begin{array}{l}x \\
x\end{array}$ & & $\begin{array}{l}x \\
x\end{array}$ & $\begin{array}{l}x \\
x\end{array}$ & $x$ \\
\hline $6 \mid R \cdot 2,46$ & VP & $F$ & 90 & & & & R & $x$ & $\mathrm{x}$ & $x$ & $\mathrm{x}$ & $\mathrm{x}$ & $x$ & & & & & & & $x$ & & $\mathrm{x}$ \\
\hline GIR-CC & vP & $R / F$ & $\begin{array}{l}100 \\
100\end{array}$ & D & $\because$ & $\mathrm{x}$ & R & $\hat{x}$ & $\hat{x}$ & $\hat{x}$ & $\hat{x}$ & $\hat{x}$ & $\hat{x}$ & & & & & & & $\therefore$ & & $\mathrm{x}$ \\
\hline $62 R-1.129-131$ & vP & $\mathrm{F}$ & 90 & D & VR & & & $\mathrm{x}$ & $\mathrm{x}$ & $\mathrm{x}$ & $\mathrm{x}$ & $\mathrm{x}$ & & & & $\mathrm{x}$ & & & & $\mathrm{x}$ & & \\
\hline $62 \mathrm{R} \cdot 2,31-33$ & VP/P & $R$ & 95 & D & VR & & & $\mathrm{x}$ & $\mathrm{x}$ & $x$ & $x$ & $\mathrm{x}$ & $\mathrm{x}$ & & & & & of & & $\hat{x}$ & of & $\mathrm{x}$ \\
\hline $62 R-3.26-28$ & $\mathrm{VP}$ & & 100 & D & VR & $x$ & $R$ & $x$ & $x$ & $x$ & $x$ & $x$ & & & & & & & , & : & : & ? \\
\hline $62 R-C C$ & vP & $F$ & 100 & & & . & $\mathrm{R}$ & $\mathrm{x}$ & $\mathrm{x}$ & $\mathrm{x}$ & $x$ & $\mathrm{x}$ & & & & & & & & & & \\
\hline $63 R-1.84-86$ & vp & $R / F$ & 100 & & VR & & $R$ & $x$ & $\mathrm{x}$ & & $x$ & $\mathrm{x}$ & & & & $\therefore$ & & & & & & \\
\hline 63R-2, 109-111 & VP & R/F & 100 & D & VR & & $\mathrm{F}$ & $x$ & $x$ & $x$ & x & $x$ & & & & $\mathrm{x}$ & & & & & & \\
\hline $\begin{array}{l}63 R-3.301-103 \\
63 R-4.57-59\end{array}$ & $\begin{array}{l}\text { VP } \\
\text { VP/P }\end{array}$ & & $\begin{array}{r}100 \\
90\end{array}$ & $\begin{array}{l}\mathrm{D} \\
\mathrm{D}\end{array}$ & $\begin{array}{l}\mathrm{VR} \\
\mathrm{VR}\end{array}$ & $x$ & & $\begin{array}{l}x \\
x\end{array}$ & $\begin{array}{l}x \\
x\end{array}$ & $\begin{array}{l}x \\
x \\
x\end{array}$ & $\begin{array}{l}x \\
x \\
x\end{array}$ & $\begin{array}{l}x \\
x \\
x\end{array}$ & $x$ & & $\mathrm{x}$ & $x$ & $\mathrm{x}$ & & & $x$ & $x$ & $\mathrm{x}$ \\
\hline 63R-CC & vP & $R$ & 90 & D & VR & & & $x$ & $x$ & $x$ & $x$ & $x$ & $\mathrm{x}$ & $\mathrm{x}$ & & & & of & & & & \\
\hline $64 R-1,64-66$ & vp & $F$ & 90 & D & $R$ & $\mathrm{x}$ & $\mathrm{F}$ & $\ddot{x}$ & $\mathrm{x}$ & $x$ & $x$ & $x$ & $\mathrm{x}$ & & & $x$ & $x$ & & & & of & \\
\hline $64 R-2,7-9$ & vP & R & 100 & D & VR & & F & $\mathrm{x}$ & $\mathrm{x}$ & $\mathrm{x}$ & $\mathrm{x}$ & $\mathrm{x}$ & & & & $\mathrm{x}$ & $\mathrm{x}$ & & & & & \\
\hline $\begin{array}{l}64 R-3,18-20 \\
64 R \cdot C C\end{array}$ & $\begin{array}{l}\text { VP } \\
\text { VP }\end{array}$ & $\begin{array}{l}R \\
F\end{array}$ & $\begin{array}{l}100 \\
100\end{array}$ & $\begin{array}{l}\text { D } \\
\text { D }\end{array}$ & $\begin{array}{l}R \\
\text { VR }\end{array}$ & of & F & $\begin{array}{l}x \\
x\end{array}$ & $\begin{array}{l}x \\
x\end{array}$ & $\begin{array}{l}x \\
x\end{array}$ & $\begin{array}{l}x \\
x\end{array}$ & $\begin{array}{l}x \\
x\end{array}$ & $\mathrm{x}$ & & of & $\mathrm{x}$ & & & & : & & $x$ \\
\hline $65 R-1.56-57$ & vp & & 100 & D & $\mathrm{VR}_{\mathrm{N}}$ & & $F$ & $x$ & $x$ & $x$ & $x$ & $x$ & & & $x$ & & & & & & & \\
\hline $65 \mathrm{R}-1.144-145$ & $\mathrm{vP}$ & R/F & 90 & & VR & & VR & $\hat{x}$ & $\hat{x}$ & $\hat{x}$ & $\hat{x}$ & $\hat{x}$ & & & $\hat{\text { of }}$ & & & & & & & \\
\hline $65 \mathrm{R}-2,22-23$ & VP & $R / F$ & 100 & D & VR & & $R$ & $\mathrm{x}$ & $\mathrm{x}$ & $\mathrm{x}$ & $\mathrm{x}$ & $x$ & of & & & $x$ & & & & of & & \\
\hline $65 \mathrm{R}-2.119-120$ & vp & R/F & 90 & & $R$ & & R & $x$ & $\mathrm{x}$ & $\hat{x}$ & $\mathrm{x}$ & $\hat{x}$ & $\mathrm{x}$ & & & & & & & & & $x$ \\
\hline 65R-CC & VP & & & & & $\mathrm{x}$ & R & & $\mathrm{x}$ & & & & of & . & 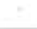 & & & $\because$ & & $\cdot$ & . & $x$ \\
\hline 66R-1, 99-101 & vP & R & 100 & & $\mathbf{R}$ & $\mathrm{x}$ & FIC & $\mathrm{x}$ & $\mathrm{x}$ & $\mathrm{x}$ & $\mathrm{x}$ & $\mathrm{x}$ & & & & & & & & $\mathrm{x}$ & & \\
\hline $66 R \cdot 3,58-60$ & VP & VR & 100 & & $R$ & $\mathrm{x}$ & F/C & $x$ & × & 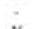 & $\mathrm{x}$ & $x$ & & & & & & & & & & 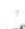 \\
\hline $\begin{array}{l}\text { 66R-CC } \\
67 \mathrm{R}-46-48\end{array}$ & vp & VR & 100 & & VR & $x$ & F/C & $x$ & $\mathrm{x}$ & $x$ & $*$ & $x$ & & & & & & & & & & cf \\
\hline $\begin{array}{l}67 R-1.46-48 \\
67 R-2,46-48\end{array}$ & $\begin{array}{l}\text { VP } \\
P\end{array}$ & $\begin{array}{l}\mathrm{R} \\
\mathrm{F}\end{array}$ & $\begin{array}{r}100 \\
90\end{array}$ & D & $\begin{array}{l}R \\
R\end{array}$ & $\begin{array}{l}x \\
x\end{array}$ & & $x$ & $\mathrm{x}$ & $\mathrm{x}$ & $\begin{array}{l}x \\
x\end{array}$ & $\begin{array}{l}x \\
x\end{array}$ & & & & & & & & & cf & $\mathrm{x}$ \\
\hline $67 R-3.46-48$ & vp & $\mathrm{R} / \mathrm{F}$ & 100 & D & $\mathrm{R}$ & $\mathrm{x}$ & VR & $\mathrm{x}$ & $\mathrm{x}$ & $\mathrm{x}$ & $\mathrm{x}$ & $\mathrm{x}$ & ef & & ef & & & & & & cf & $\mathrm{x}$ \\
\hline $67 R-4,102-104$ & vp & R & 100 & D & $\mathrm{R}$ & $x$ & $R / F$ & $x$ & $\mathrm{x}$ & $\mathrm{x}$ & $\mathrm{x}$ & $x$ & & & & . & & & & & & \\
\hline $67 R-5,46-48$ & vP & $R$ & 100 & & VR & & F/C & & & $x$ & $x$ & $x$ & & & & $\therefore$ & & & & & & \\
\hline 67R-CC & VP & $\mathrm{VR/R}$ & 100 & & Q & & $R / F$ & $\mathrm{x}$ & & & $x$ & $\mathrm{x}$ & & & & $x$ & & & & & & \\
\hline $68 R-1,85-87$ & & & & & VR & & & & $=$ & & & & & & & & & & & & 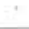 & $x^{2}+2$ \\
\hline $68 R$ & & $\mathbf{R}$ & 10 & D & VR & $\mathrm{x}$ & F & & $\mathrm{x}$ & . & $x$ & $x$ & & & & & & & & & & \\
\hline & & R & & & $R$ & & & & $x$ & of & $\mathrm{x}$ & $\mathrm{x}$ & of & & & & & & 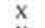 & & $x$ & \\
\hline 68 & vi & $\mathbf{R}$ & 100 & D & $\mathrm{R}$ & & $\mathbf{R}$ & $\boldsymbol{X}$ & $\mathrm{x}$ & $\mathrm{x}$ & $\mathrm{x}$ & & & & & & & & 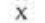 & & & \\
\hline $69 \mathrm{R}-1,44-46$ & vp & $R_{R}^{R}$ & 100 & & VR & & is & & $\begin{array}{l}x \\
x\end{array}$ & $x$ & $x$ & & $\therefore$ & & & & $\therefore$ & & $x$ & is & & \\
\hline $69 \mathrm{R}-2,45-47$ & & R & & & & & & & & & & $x$ & & & & & & & & & & \\
\hline $69 R-3,17-19$ & VP & & 90 & D & R & $x$ & $R / F$ & $\mathrm{x}$ & $\mathrm{x}$ & $\mathrm{x}$ & $\mathrm{x}$ & $\mathrm{x}$ & & & $\mathrm{x}$ & $\mathrm{x}$ & of & of & & & & . \\
\hline $\begin{array}{l}69 R-4.16-18 \\
69-5\end{array}$ & VP & $\underset{F}{R / F}$ & 100 & & ${ }_{R}^{R}$ & $\mathrm{x}$ & ${ }_{R}^{R}$ & $\begin{array}{l}x \\
x\end{array}$ & $\therefore$ & ef & $\begin{array}{c}x \\
\text { of }\end{array}$ & $\stackrel{x}{x}$ & & & : & & & & & & & \\
\hline $69 \mathrm{R}-\mathrm{CC}$ & $\mathrm{VP}$ & $F$ & 100 & & & & $\hat{F}$ & $\hat{n}$ & & $\hat{x}$ & $\mathrm{x}$ & $\hat{x}$ & $\mathrm{x}$ & & & $\mathrm{x}$ & & & & & & \\
\hline $70 \mathrm{R}-1.78-80$ & vp & $\mathrm{R}$ & 100 & & VR & & R & $\mathrm{x}$ & $\mathrm{x}$ & $\mathrm{x}$ & $\mathrm{x}$ & $\mathrm{x}$ & & & & $\hat{x}$ & & & & & & \\
\hline $70 R \cdot 2.62-64$ & VP & & & & VR & & $\mathbf{F}$ & & & $\mathrm{x}$ & $\mathrm{x}$ & $\mathrm{x}$ & & & & & & & & & & . \\
\hline $70 R-3.144-146$ & vP & R & & & VR & & - & & $x$ & $\hat{x}$ & $\hat{x}$ & $\hat{x}$ & & & & & & & & & & \\
\hline $70 R-4,44-46$ & vp & R & 100 & & & . & R & $\mathrm{x}$ & & $\mathrm{x}$ & $\mathrm{x}$ & $\mathrm{x}$ & & & . & $\mathrm{x}$ & of & & & & & \\
\hline $\begin{array}{l}70 \mathrm{R}-\mathrm{CC} \\
71 \mathrm{R}-1,43-45\end{array}$ & $\begin{array}{l}\text { VP } \\
\text { vP }\end{array}$ & $\begin{array}{c}\text { VR } \\
\text { VR/R }\end{array}$ & $\begin{array}{l}100 \\
100\end{array}$ & & & $x$ & VR & $x$ & $\mathrm{x}$ & $\begin{array}{l}x \\
x\end{array}$ & $\begin{array}{l}x \\
x\end{array}$ & $\begin{array}{l}x \\
x\end{array}$ & & & . & & & & & & & \\
\hline $71 R-1,86-88$ & $v v_{t}-2-1$ & & & & VR & $\mathrm{x}$ & & $\mathrm{x}$ & $\mathrm{x}$ & of & & $\mathrm{x}$ & & & of & & & & & & & \\
\hline $71 \mathrm{R}-1.14$ & $\mathrm{v}$ & $\hat{R}$ & & & & & $\hat{R}$ & & & of & $\hat{x}$ & & & 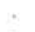 & & & & & & & & \\
\hline $71 R-2.52-54$ & vp & $\kappa$ & 100 & & & & c & & & . & $\hat{x}$ & $\mathrm{x}$ & & & & & & & & & & \\
\hline $\begin{array}{l}7 \mid R-2,59-61 \\
7 \mid R-2,125-127\end{array}$ & vp & VR & 95 & i & VR & & & & & & $x$ & $\mathrm{x}$ & of & if & & & & & & & & \\
\hline & & & & & & & & & & & & & & & & & & & & & & \\
\hline $\begin{array}{l}718-3,31-33 \\
718-3\end{array}$ & $\begin{array}{l}\text { vp } \\
\text { yp }\end{array}$ & ${ }_{R}^{R}$ & 100 & & $\begin{array}{l}\text { VR } \\
\text { vR }\end{array}$ & of & $\mathrm{F}$ & $\begin{array}{l}x \\
x \\
x\end{array}$ & if & $\begin{array}{l}x \\
x\end{array}$ & $\begin{array}{l}x \\
x\end{array}$ & & $\begin{array}{l}x \\
x\end{array}$ & & & $\therefore$ & & & & & & \\
\hline & vp & & & & & : & A & & & & & & & & & & & & & & & \\
\hline TIR-CC & & & & & & & & & & & & & & & & & & & & & & \\
\hline
\end{tabular}

Notes: Abbreviations as in Table $\mathrm{I}$, with the addition of the following: Preservation: $\mathrm{P}=$ poor. Abundance: $\mathrm{A}=$ abundant. Dissolved: $\mathrm{D}=$ dissolved and/or distorted larger planktonic foraminifers. An asterisk $(*)$ indicates thin section cut horizontal to bedding. 
Table 2 (continued).

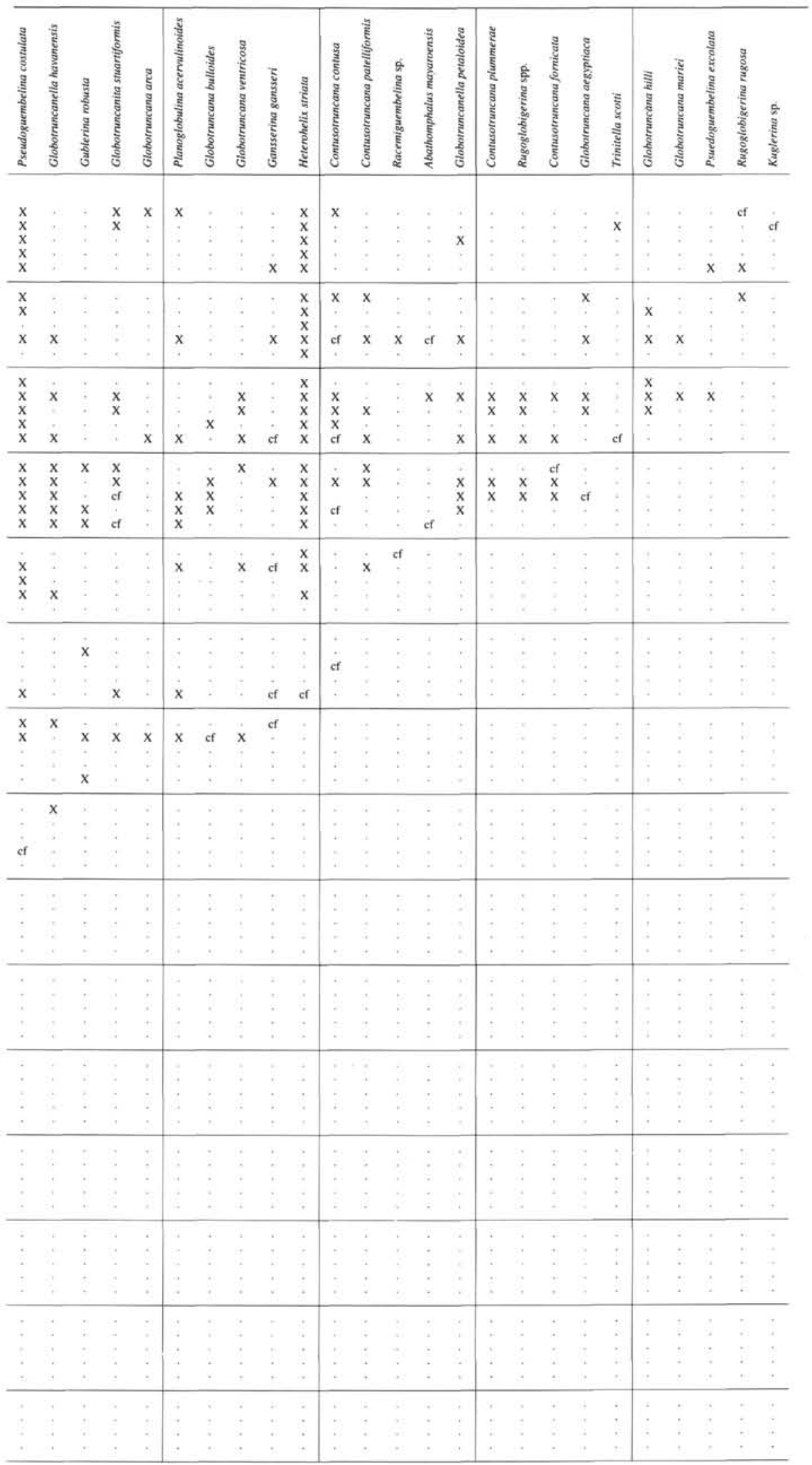




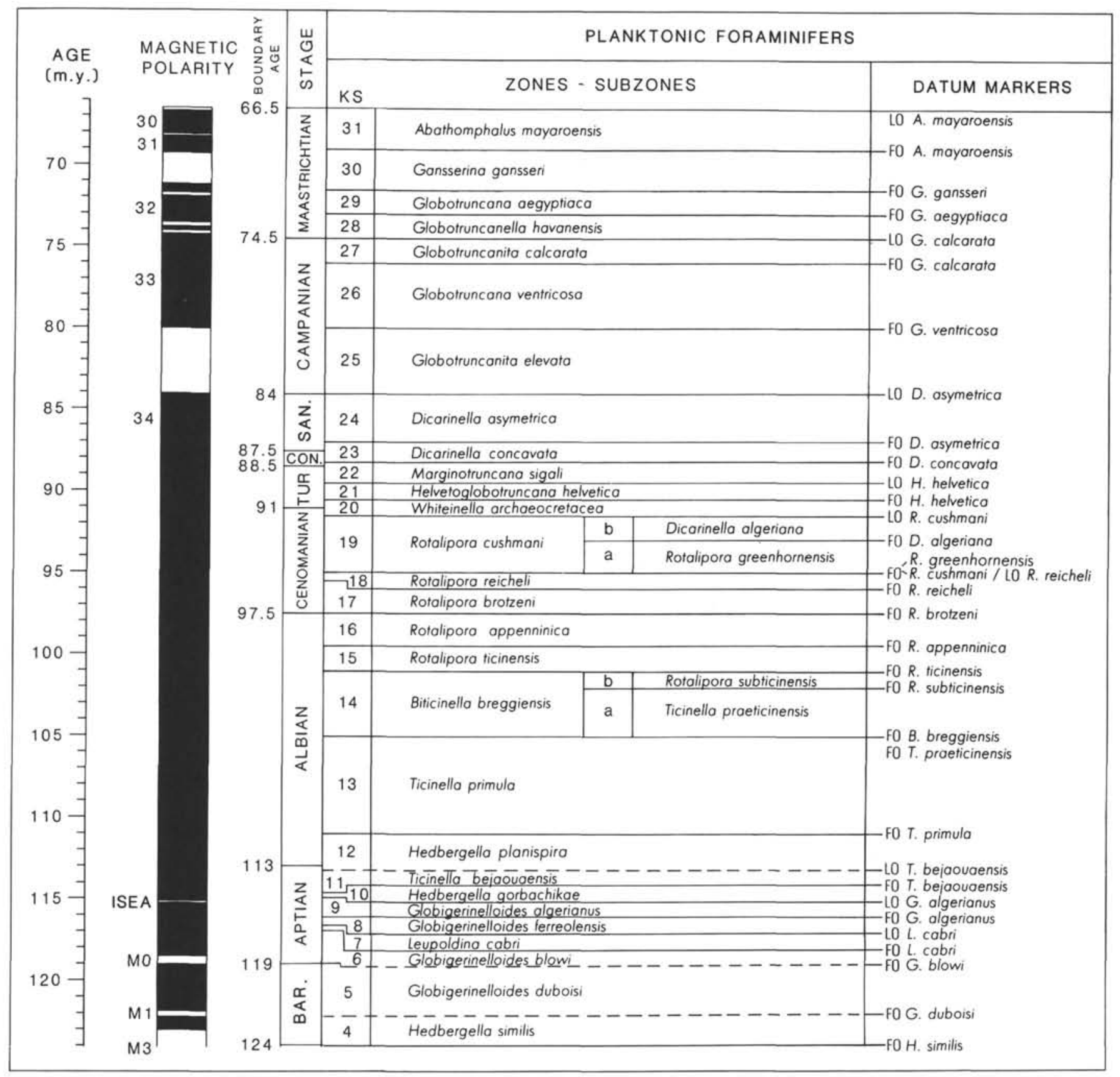

Figure 2. Zonation for Cretaceous planktonic foraminifers examined in thin section (modified from Sliter, 1989; Leckie and Bralower, 1991). Magneto-geochronology after Harland et al. (1982) and Kent and Gradstein (1985). KS = Cretaceous zone notation. $\mathrm{F}=$ first occurrence, and L = last occurrence. ISEA = reversed polarity site of Vandenberg et al. (1978).

from the interbedded claystone and siltstone in Cores $130-807 \mathrm{C}-73 \mathrm{R}$ and $-72 \mathrm{R}$ are limited to mostly common, recrystallized radiolarians of early Aptian age (Takahashi and Ling, this volume).

\section{Correlation}

Sites 288 and 289, drilled during DSDP Leg 30, recovered Aptianaged sediment from Ontong Java Plateau and are shown in Figure 4. Ages based on planktonic foraminifers examined in thin section previously were described by Sliter (1992). Here, we briefly reexamine these data and compare the results to those from Leg 130. At Hole $288 \mathrm{~A}$, drilled in $3000 \mathrm{~m}$ of water on the southeastern edge of the plateau (Fig. 1), the deepest core drilled before the site was abandoned ( $3988.5 \mathrm{~m}$ below present sea level) contains late Aptian planktonic foraminifers from the Ticinella bejaouaensis Zone in Sample 30288A-30-1, 81-82 cm. The lithology of the core consists of light grey, laminated limestone interbedded with chert. Similar limestone from the base of Core 30-288A-29 contains foraminifers from the late Albian Rotalipora appenninica Zone in Sample 30-288A-29-1, 136-139 cm. Separating these two samples is a hiatus of over 15 m.y. (Fig. 2).

Site 289 was drilled on top of the plateau at a water depth of $2206 \mathrm{~m}$. Basaltic basement was encountered in Core 30-289-132 ( $3468.5 \mathrm{~m}$ below present sea level) overlain by tuff and limestone. Planktonic foraminifers from the base of the yellowish brown lime- 


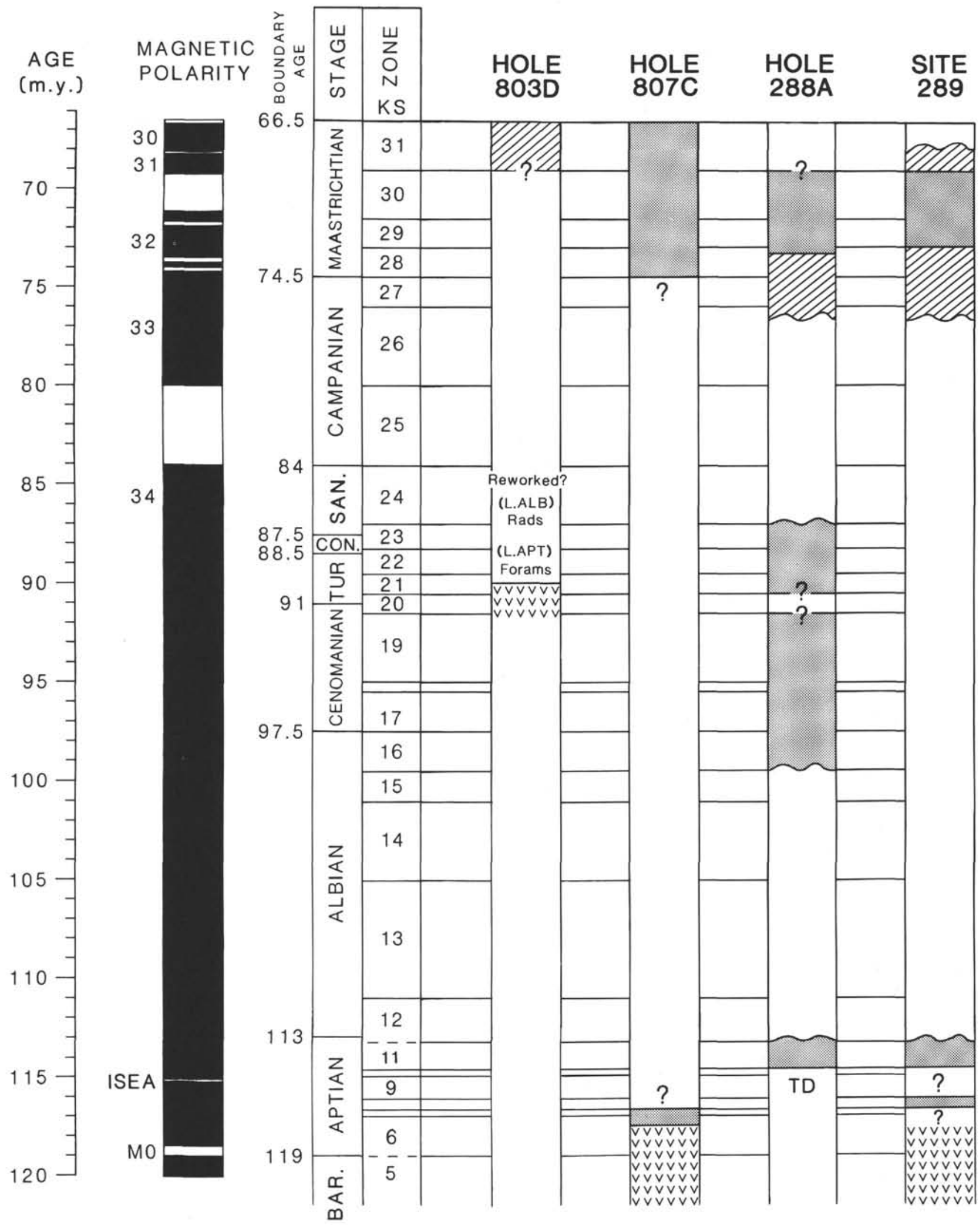

Figure 3. Age of Cretaceous sediment recovered from the Ontong Java Plateau. Data from planktonic foraminifers (stipple pattern) and calcareous nannofossils (diagonal pattern) shown for site or specific hole at site. Cenomanian to Maastrichtian age data for planktonic foraminifers and calcareous nannofossils modified from descriptions in the Initial Reports of the DSDP. Zone assignment based on correlation to zonation of Figure 2. Basement age shown by " $v$ " pattern. Wavy lines $=$ unconformities. TD $=$ total depth (hole abandoned before reaching basement). 


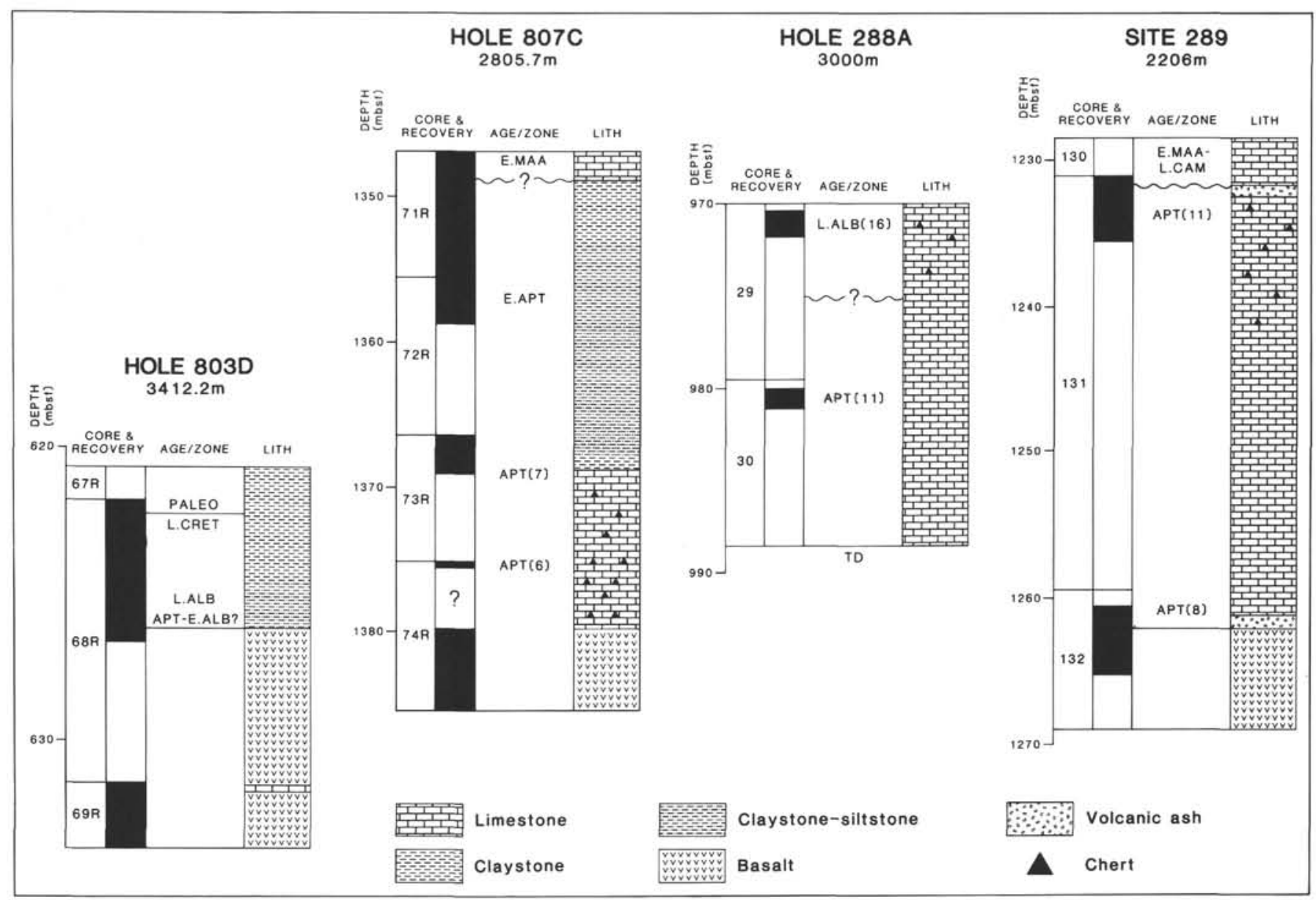

Figure 4. Age of oldest sediments from Ontong Java Plateau. Black area in core column shows amount of sediment recovered for each core. Cretaceous zone notation (KS, see Fig. 2) is used in Age/Zone column. Lithology filled in between recovered intervals. TD = total depth.

stone overlying the tuff in Sample $30-289-132-2,37-40 \mathrm{~cm}$, are placed in the late Aptian Globigerinelloides ferreolensis Zone (Sliter, 1992). Although the assemblage is very similar in species composition to those of Hole $807 \mathrm{C}$ in Section 130-807C-73-2, the presence of Leupoldina reicheli indicates a somewhat younger age. Whole specimens from Sample 30-289-132-1, 21-23 cm, include Globigerinelloides blowi, G. gottisi, Hedbergella hispaniae, and $H$. praetrocoidea and likewise are assigned to the Globigerinelloides ferreolensis Zone. Core 30-289-131 consists of two sections of laminated limestone and interbedded chert overlain by an interval of reddish brown, zeolite-bearing tuff followed in turn by white limestone at the top of the core. Calcareous nannofossils from the white limestone yield a late Campanian to early Maastrichtian age (Shafik, 1975), whereas upper Aptian planktonic foraminifers were reported from the laminated limestone below the reddish brown tuff in Sample 30-289-131-1, 145-146 cm (Andrews, Packham, et al., 1975). The presence of Aptian sediments is confirmed by the recovery of both Ticinella bejaouaensis and Hedbergella trocoidea as whole specimens in Sample 30-289-131-3, 80-82 cm, which are assigned to the Ticinella bejaouaensis Zone of latest Aptian age.

\section{Late Cretaceous}

\section{Hole $807 \mathrm{C}$}

Late Cretaceous planktonic foraminifers from Hole $807 \mathrm{C}$ first appear in the pale green to light brown limestone with claystone interbeds of Core 130-807C-71R just above the contact with the underlying claystone and siltstone unit (see Table 2 and summary in
Fig. 5). The basal limestone in Sections $130-807 \mathrm{C}-71 \mathrm{R}-2$ and -3 contains numerous thin laminae of reddish brown claystone that become less common upsection through Section 130-807C-70R-3. Above this interval, the limestone is mostly white, homogeneous, and bioturbated through Core $130-807 \mathrm{C}-59 \mathrm{R}$ and then is distinctly laminated through Core 130-807C-55R. Assemblages of planktonic foraminifers below Core 130-807C-64 consist of rare, poorly preserved, minute specimens with occasional ghosts of larger specimens (Fig. 6). Larger planktonic foraminifers become increasingly common upsection and then decrease in abundance near the Cretaceous/Tertiary boundary in Core 130-807C-55R. It is important to note that ages based on the larger planktonic foraminifers, and perhaps all the planktonic species, must be considered minimal ages because of the pervasive dissolution and common resedimentation and/or reworking throughout the cored interval.

The age of the basal limestone and claystone unit is difficult to determine due to the prevalence of small specimens of Hedbergella and Globigerinelloides that continue as the background fauna throughout the Upper Cretaceous section, as well as to the rarity and poor preservation of larger planktonic foraminifers (Table 2). Species present include Globigerinelloides messinae, G. subcarinatus, Heterohelix glabrans, small specimens of Pseudotextularia elegans and several hedbergellids that range throughout the Campanian and Maastrichtian. The best age information is derived from several poorly preserved, larger planktonic foraminifers. A specimen questionably identified as Globotruncanita stuarti was found in Sample 130-807C$71 \mathrm{R}-2,125-127 \mathrm{~cm}$. The presence of this species is important as it first occurs at the Campanian/Maastrichtian boundary. Somewhat 


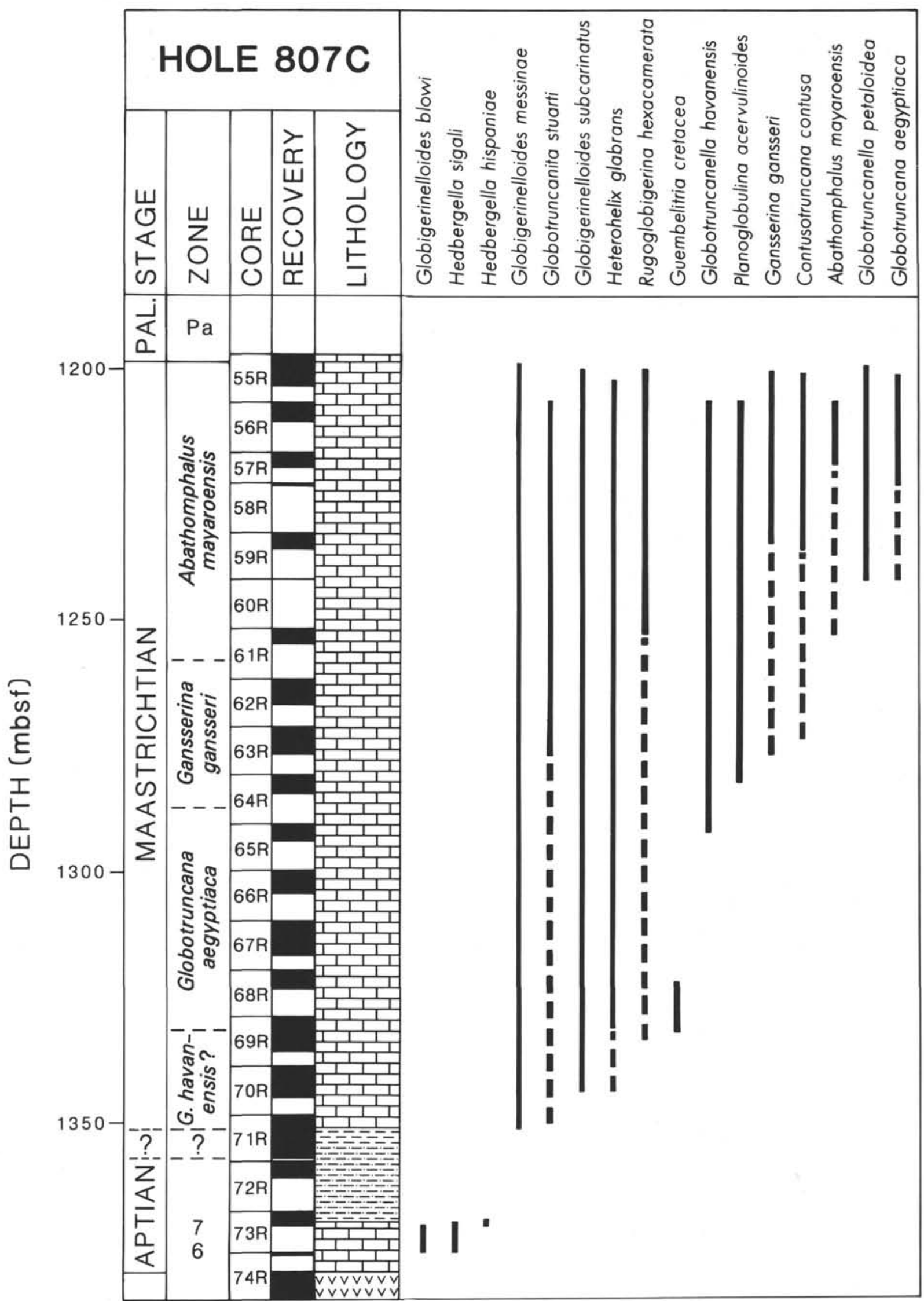

Figure 5. Ranges of stratigraphically significant Cretaceous planktonic foraminifers from Hole 807C. Cretaceous zone notation (KS) used for Aptian planktonic foraminiferal zones. Dashed line = probable identification. 


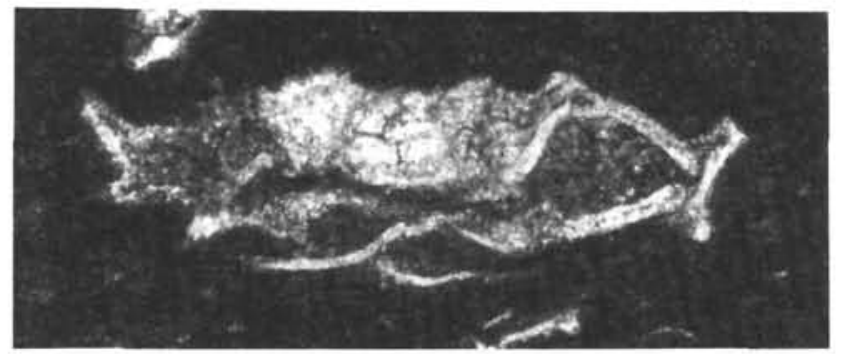

A

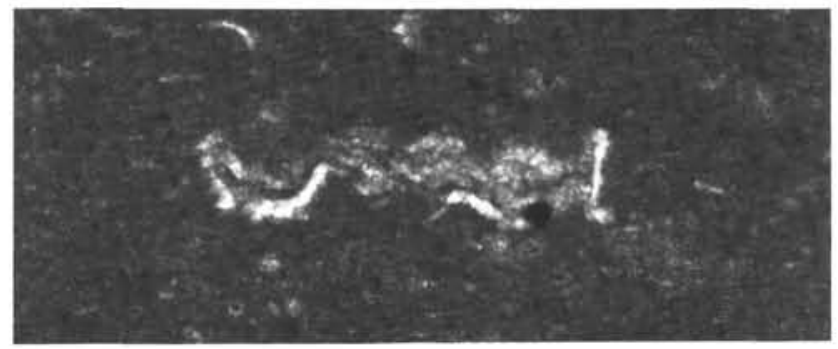

B

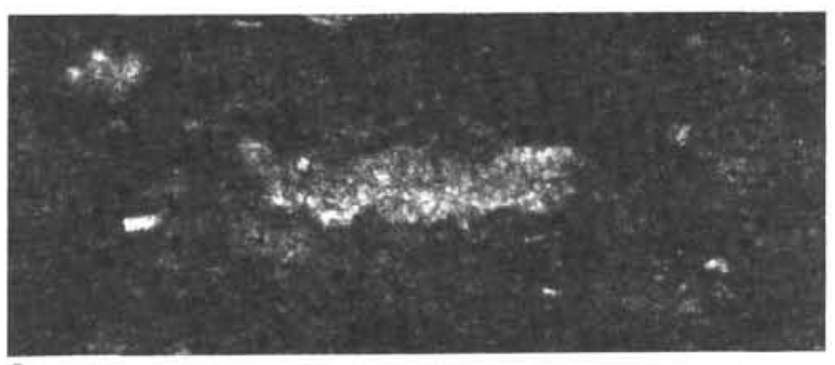

C

Figure 6. Photomicrographs of Cretaceous planktonic foraminifers illustrating progressive stages of carbonate dissolution, Hole 807C; Sample 130-807C$59 \mathrm{R}-2,99-100 \mathrm{~cm}$, all $150 \times(3 \mathrm{~cm}=200 \mu \mathrm{m})$. A. Collapsed and partially dissolved foraminifer. B. Partial ghost of foraminifer with small portions of wall remaining. C. Ghost of planktonic foraminifer.

higher in Core 130-807C-69R, a specimen is questionably assigned to Rugoglobigerina hexacamerata, which first occurs in the Globotruncana aegyptiaca Zone. These specimens suggest that the basal unit is most likely early Maastrichtian in age. Lending support to this age interpretation is the FO of the calcareous nannofossils Quadrum gothicum in Section 130-807C-71R-3 and Q. trifidium between Samples 130-807C-71R-1, 37-38 cm, and -70R-CC, which indicate a late Campanian to early Maastrichtian age range for the basal unit (Kroenke, Berger, Janecek, et al., 1991).

The first influx of larger planktonic foraminifers occurs in Cores 130-807C-64R to -63R with Globotruncanella havanensis and Globotruncanita stuarti, the FO of Planoglobulina acervulinoides, and questionable Contusotruncana contusa and Gansserina gansseri. The rarity, poor preservation, and small size of the "larger" planktonic foraminifers makes the zonal assignment difficult. The species present, however, indicate a middle Maastrichtian age for this interval, probably within the Gansserina gansseri Zone.

The major influx of large planktonic foraminifers in Cores 130-807C$61 \mathrm{R}$ to $-55 \mathrm{R}$ is accompanied by Contusotruncana contusa, C. plummerae, Gansserina gansseri, Globotruncana aegyptiaca, Globotruncanella petaloidea, Pseudoguembelina excolata, Racemiguembelina sp., Rugoglobigerina hexacamerata, and questionable Abathomphalus mayaroensis, among others. This assemblage yields a late Maastrichtian age that we assign to the Abathomphalus mayaroensis Zone.
The Cretaceous/Tertiary boundary was placed at the top of Core 130-807C-55R between Samples 130-807C-55R-1, 49-50 cm, and $-55 \mathrm{R}-1,35-36 \mathrm{~cm}$ (see Premoli Silva, this volume). Thin sections from both samples are cut parallel to bedding. Although this orientation increases the number of axial or spiral views in the section and thus aids the identification of minute Paleocene species, at the same time it increases the chance of missing an important layer in this laminated interval. Nevertheless, the first Paleocene specimens attributed to Eoglobigerina fringa and elongate heterohelicids that resemble Chiloguembelina occur in Sample 130-807C-55R-1, 35-36 cm, within an assemblage of small- to medium-sized Cretaceous planktonic species of Globigerinelloides, Heterohelix, Pseudotextularia, and Hedbergella listed in order of abundance. Above this sample, an interval of strong dissolution flanks a thin ash layer (Sample 130$807 \mathrm{C}-55 \mathrm{R}-1,27 \mathrm{~cm}$ ) and is followed by a flood of small, globular Cretaceous planktonic species in samples from the top of the core. This placement of the Cretaceous/Tertiary boundary differs from the shipboard description that was based on the elevated abundance of the calcareous dinoflagellate Thoracosphaera in Section 130-807C54R-3 (Kroenke, Berger, Janecek, et al., 1991).

\section{Correlation}

Correlation of the Upper Cretaceous section from Hole 807C to similar sections from holes at other sites on Ontong Java Plateau is problematic (Figs. 3-4). At Hole 803D, the Upper Cretaceous sequence is contained within the largely noncalcareous claystone and siltstone unit. Calcareous nannofossils from the top of the claystone and siltstone unit define the upper Maastrichtian and an apparently intact but condensed Cretaceous/Tertiary boundary sequence (Kroenke, Berger, Janecek, et al., 1991).

At Site 288, the presence of planktonic foraminifers distinguishes part of the early Maastrichtian, the middle Maastrichtian Gansserina gansseri Zone, and places the Cretaceous/Tertiary boundary in the uncored interval between Cores 30-288A-8 and -9 (Andrews, Packham et al, 1975). Calcareous nannofossils possibly extend the record into the late Campanian (Shafik, 1975). A similar record was found at Site 289; however, the Cretaceous/Tertiary boundary reported from Core 30-289-30 is missing part of both the uppermost Maastrichtian and basal Paleocene (Andrews, Packham, et al., 1975).

\section{PALEOENVIRONMENT}

Interpretation of the Cretaceous depositional environments represented by the Leg 130 sediments is based on the following criteria: (1) the taxonomic composition of the foraminiferal assemblages, (2) the presence of other biogenic constituents, (3) preservation of the foraminifers and radiolarians, (4) lithology, and (5) sedimentary structures. In addition to interpreting the paleobathymetric record, these criteria are used to determine the position of the lysocline and carbonate compensation depth (CCD), as well as evidence of resedimentation and reworking. The goal is recognition of chemical or mechanical processes that may have removed or enriched foraminiferal species, thereby affecting the paleoecologic and biostratigraphic interpretation of the foraminiferal assemblages.

In the following discussion, we use the terms "bathyal" and "abyssal" in the bathymetric interpretations. These terms refer to the stratified character of both the modern and Cretaceous world ocean that is reflected in the bathymetric distribution of the biofacies and the lithofacies. Here we use water depths from 200 to $3500 \mathrm{~m}$ for the bathyal zone and depths greater than $3500 \mathrm{~m}$ for the abyssal zone. The boundary between the bathyal and abyssal realms essentially defines the top of the CCD in mid-latitude oceans today and is expressed by the transition between calcareous and noncalcareous deposits. Here we recognize the boundary by the loss of calcareous species and the appearance of numerous agglutinated species. 
The bathymetric position of the $\mathrm{CCD}$ and the associated dissolution gradient obviously are affected by ocean productivity, deep-water circulation, and climatic conditions that have varied through time. For example, Thierstein (1979) suggests that the CCD in the Cretaceous Pacific Ocean may have fluctuated between paleodepths of $>6 \mathrm{~km}$ to $<2.5 \mathrm{~km}$. Furthermore, these data indicate that during deposition of the claystone and siltstone units of Sites 803 and 807, the CCD stood as high or higher than $3 \mathrm{~km}$. Fluctuations of the CCD likewise may have compressed the bathymetric subdivisions of the bathyal zone that are defined by the boundaries of specific water masses. In our interpretations, we use $200-500 \mathrm{~m}$ for the upper bathyal zone, 500 $1500 \mathrm{~m}$ for the middle bathyal zone, and $1500-3500 \mathrm{~m}$ for the lower bathyal zone.

Analyses of the Leg 130 material show that the Cretaceous sediments were deposited in the lower bathyal to abyssal zones and were strongly influenced by diagenesis and resedimentation processes. No shallow-water biogenic material such as orbitolinids, bivalves, echinoid fragments, or calcareous algae were recovered.

\section{Aptian-Albian Environments}

\section{Hole 803D}

Cretaceous foraminifers from the claystone unit in Hole 803D are limited to two samples. The first assemblage from Sample 130-803D68R-3, 143-145 cm, consists of a dominantly agglutinated fauna with very rare, poorly preserved, calcareous species. Present are species of Glomospirella, Haplophragmoides, Pseudobolivina, and Trochammina that, together with the lithology, indicate deposition at abyssal water depths below the CCD. The occurrence of this assemblage in an interval characterized by minor radiolarian-rich layers and millimeter-scale laminae suggests that the calcareous foraminifers were introduced by mass-flow processes (i.e., slow to rapid gravitational processes). This hypothesis is supported by the second assemblage from Sample 130-803D-68R-1, 110-112 cm, which comes from an interval of bioturbated claystone. The abyssal assemblage is totally agglutinated and, in addition to the above taxa, includes species of Ammodiscus and Paratrochamminoides. As noted earlier, however, these abyssal sediments probably were not the earliest sediments deposited on the basalt. The presence of several thin interpillow limestone fragments suggest an initial phase of carbonate sedimentation above the CCD similar to that of Site 807. All evidence of more continuous sedimentation, however, was removed during the presumed hiatus at the basalt/sediment contact.

\section{Hole 807 C}

Biogenic assemblages from the Aptian limestone of Hole 807C consist of rare planktonic and benthic foraminifers associated with very rare radiolarians and fish debris (Table 1). Benthic foraminifers, distributed homogeneously within the bioturbated limestone, are represented by flattened agglutinated tubes resembling Bathysiphon, complex-coiled agglutinated forms resembling Plectorecurvoides, and calcareous forms that include small, narrow specimens of Dentalina, other rare nodosariids, and Gavelinella. This assemblage, especially with the number and kind of agglutinated foraminifers, is indicative of the lower bathyal zone above the $\mathrm{CCD}$ and a water depth of about $3 \mathrm{~km}$.

Several trends were noticed in the Aptian limestone sequence. An upward decrease is present in the abundance and preservation of the foraminifers approaching the overlying claystone and siltstone unit. This is seen in the number of ghosts of planktonic foraminifers, which increase from $40 \%$ at the base to $80 \%$ at the top (Table 1). An upward increase is also present in the reddish brown claystone layers (Fig. 7A); fine, brown-colored anastomosing fractures; and brownish mottled areas. These are interpreted to represent microstylolytic dissolution seams, healed tensional fractures, and the diagenetic alteration of burrowed micrite, respectively, caused by the secondary

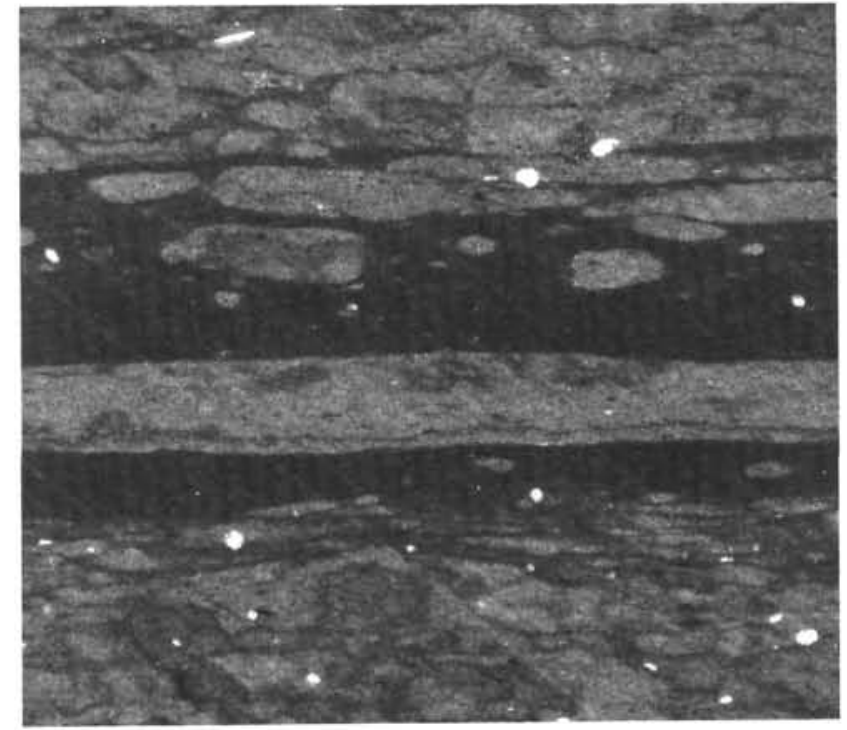

A

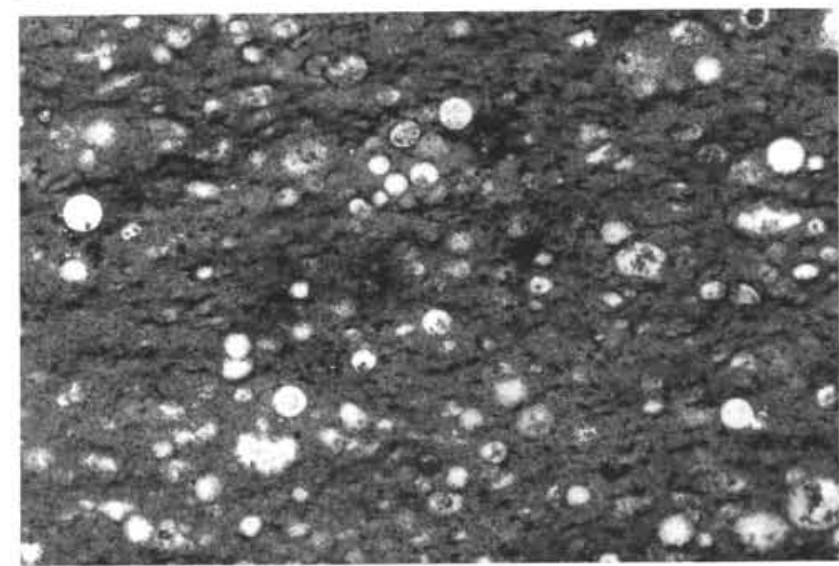

B

Figure 7. Photomicrographs of Aptian limestone, and radiolarian-rich claystone and siltstone unit of Hole $807 \mathrm{C}$; both $30 \times(3 \mathrm{~cm}=1 \mathrm{~mm})$. A. Micritic limestone with reddish brown, clay-rich solution seams possibly caused by chemical compaction, Hole $807 \mathrm{C}$, Sample 130-807C-73R-CC. White dots in both the limestone and clay-rich seams represent Cretaceous foraminifers. B. Abundant, recrystallized radiolarians in claystone and siltstone unit, Sample $130-807 \mathrm{C}-73 \mathrm{R}-1,113-115 \mathrm{~cm}$.

effects of diagenesis and chemical compaction. Supporting this interpretation is the equal distribution of planktonic foraminifers and agglutinated and calcareous benthic foraminifers in both the limestone and the brown mottled areas, and the equal or increased abundance of both planktonic and benthic foraminifers in the claystone layers. The latter case points to the differential removal of micrite before the calcareous, although altered, tests of the foraminifers.

A further trend was observed in the increased abundance of radiolarians that extends from the top of the limestone into the lower part of the claystone and siltstone unit (Fig. 7B). Within the claystone and siltstone unit, radiolarians are restricted mostly to the lighter colored siltstone interbeds, some of which are internally graded with sharp or scoured contacts, whereas the claystone contains only very rare ghosts of radiolarians, rare fish debris, and volcanic glass. The presence of the radiolarian-rich siltstone interbeds records the importance of episodic mass transport events. Sample 130-807C-71R-3, 57-59 cm, from the top of the claystone and siltstone unit, contains an exclusively agglutinated fauna and rare fish debris. Benthic foraminiferal taxa include 
Bathysiphon, Rhabdammina, Saccammina, Ammodiscus, Haplophragmoides, Trochamminoides, and Pseudobolivina.

These trends, combined with the assemblage analysis, record the transition from an initial depositional environment beneath the foraminiferal lysocline but above the $\mathrm{CCD}$ to a position beneath the $\mathrm{CCD}$. The transition was caused most likely by the subsidence of the depositional environment as the thermally elevated plateau cooled and contracted. Alternatively, the transition could have been related to the elevation of the $\mathrm{CCD}$ with rising global sea level during the mid- and Late Cretaceous or to changes in ocean productivity over the plateau during the late Albian. Albian to Cenomanian shallowing of the CCD, however, is not supported by carbonate data from the Pacific Ocean (Thierstein, 1979), and information on productivity fluctuations in the mid-latitude Cretaceous Pacific Ocean perhaps caused by changes in ocean circulation or plate motion is lacking. It is intriguing to note, however, that the increase in radiolarian abundance, although linked to increased dissolution in the Aptian at Site 807 , may also suggest changes in ocean productivity at this time.

\section{Late Cretaceous Environments}

\section{Hole $807 \mathrm{C}$}

Upper Cretaceous sedimentation at Site 807 records the reoccurrence of environmental conditions favorable to the deposition and preservation of pelagic carbonate. The micritic limestone sequence is divided into three major microfacies: (1) a short initial interval of pale green to light brown limestone with interbeds and laminae of reddish brown claystone, in which foraminifers are strongly dissolved, subhorizontal in distribution, and interspersed with layers or zones of poorly preserved radiolarians; (2) a long, largely homogeneous interval of white limestone with both bioturbated and nonbioturbated intervals, occasional limestone clasts and uniformly distributed foraminifers (Fig. 8); and (3) a distinctly laminated interval with winnowed foraminifers (Fig. 9) that starts abruptly in Core 130807C-58R and extends into the Paleogene.

Benthic foraminifers represent a rare but persistent faunal element in all three microfacies. The assemblages are overwhelmingly calcareous and agglutinated species are limited to very rare specimens of Dorothia, tubular forms, forms that are biserial in the final stage, and unrecognizable globular specimens. Of the calcareous species, the dominant assemblage that continues throughout consists of Aragonia (see Table 2 for distribution; Plate 4, Figs. 6-7), Nuttallinella, Gyroidinoides, and Gavelinella. Minor elements include Praebulimina, Pyramidina, small nodosariids, Osangularia, Pleurostomella, Globorotalites, and Stilostomella in descending order of abundance. The combined calcareous assemblage is indicative of lower bathyal environments at or above the $\mathrm{CCD}$ but within range of potential carbonate dissolution.

Several trends apparent in the distribution of planktonic foraminifers and radiolarians are illustrated in Figure 10 and Table 2. In general, the abundance of planktonic foraminifers increases gradually through the lower and middle Maastrichtian, reaches a maximum in the upper Maastrichtian, and declines at the Cretaceous/Tertiary boundary. Radiolarians show an inverse distribution and are more abundant in the lower and middle Maastrichtian, disappear in the upper Maastrichtian, and recur at the Cretaceous/Tertiary boundary. The most persistent planktonic foraminiferal assemblage found in all samples consists of small specimens of Hedbergella, Globigerinelloides, and Heterohelix, listed in order of abundance (Fig. 8A). Distinct pulses of larger, better preserved (i.e., whole) planktonic foraminifers first appear in Core 130-807C-64R and continue upsection. Below this core, larger planktonic foraminifers occur mostly as unrecognizable, dissolved, and/or compressed ghosts that initially are very rare (Fig. $8 \mathrm{~B}$ ) and gradually become more common and better preserved upsection (Fig. 8C).

We attribute these trends to a combination of interacting variables: depth variations of the $C C D$, variations in the mode of sedimentation, increased carbonate sedimentation, and/or changing ocean currents caused by the northward transit of the plateau. The rapid deepening of the CCD through the middle Maastrichtian, noted in much of the world ocean (Thierstein, 1979; Tucholke and Vogt, 1979), is indicated by the upward increase in preservation, abundance, and diversity of planktonic foraminifers and the decrease in radiolarians. Deposition of the upper Campanian to lower Maastrichtian portion of the sequence took place at or near the CCD, with the occurrence of larger planktonic foraminifers dependent, in part, on the ameliorating effects of carbonate resedimentation (Figs. 9A-9B). The reversal of these trends near the Cretaceous/Tertiary boundary (Figs. 9C-9D) indicates a change in carbonate productivity, sedimentation rates and, perhaps, a rise in the CCD similar to other sites in the Pacific (Thierstein, 1979).

Variations in the sedimentary processes within the homogeneous microfacies are shown by the pulses in larger planktonic foraminifers as well as by the biologic and lithologic character of the limestone. The abundance of planktonic foraminifers shows considerable fluctuation below the major pulse of larger planktonic foraminifers in Core 130-807C-61R (Fig. 10). Radiolarian abundance shows an inverse but even greater amplitude curve for the same interval. We think that these fluctuations reflect the variation in diagenetic potential of sediments derived from different accumulation processes and deposited in the lower bathyal, if not sublysoclinal, environment.

Although typically appearing "homogeneous" in thin section, the limestone in this interval often contains flattened limestone clasts of $<1 \mathrm{~mm}$ to $1 \mathrm{~cm}$ in length arranged in horizontal to subhorizontal fashion, as well as occasional laminae. In other sections, the limestone appears structureless or contains subhorizontal marbling and mottling. Further, although much of the limestone is bioturbated, the structureless intervals or those that contain clasts generally are not burrowed. Thus, the homogeneous interval records a variety of accumulation processes from rapid, in the case of the clast-bearing intervals that resulted from debris flows perhaps triggered by slumps or slides, to slow in the marbled and structureless intervals, which suggest homogenization caused by slow gravity flow or creep. These processes appear similar, if not identical, to those interpreted from the seismic profiles and box cores of near-surface sediment obtained from the plateau (Berger and Johnson, 1976; Berger et al., 1977). Accordingly, we conclude that the character of the curves shown in Figure 10 reflects the influence of sediment composition, grain size, porosity, and accumulation rates on the intensity of dissolution in the lower bathyal environment.

The distribution and preservation of the planktonic foraminifers also is related to these processes and to the history of winnowing that is unique to the plateau. We think that the paucity of larger planktonic foraminifers in the lower part of the homogeneous unit and their gradual but pulselike increase in abundance upsection is caused more by the depositional processes than by fluctuations in dissolution. The presence of dissolution-susceptible taxa such as thin-walled species of Hedbergella and Globigerinelloides (Douglas, 1971; Sliter, 1992) throughout the section and the lack of fragments of the thicker walled, more heavily ornamented larger species, regardless of differences in chemical composition and porosity of the test, indicate that differential dissolution was not the primary factor controlling the distribution of the larger planktonic foraminifers.

Instead, we attribute these differences to the history of current winnowing and resedimentation that characterizes much of the sedimentary sequence of the plateau since the mid Cretaceous (Andrews, Packham, et al., 1975; Sliter, 1992). Following this hypothesis, the pelagic carbonate in the lower part of the homogeneous interval represents a combination of reworked, fine-grained material derived from areas of current winnowing that contains the small planktonic foraminifers, and debris-flow deposits that contain the larger planktonic foraminifers. In fact, the distribution of the larger forms tends to correlate with the clast-bearing intervals. The pulses of the larger planktonic foraminifers in the middle and upper Maastrichtian presumably are derived from the increased sedimentation of pelagic 


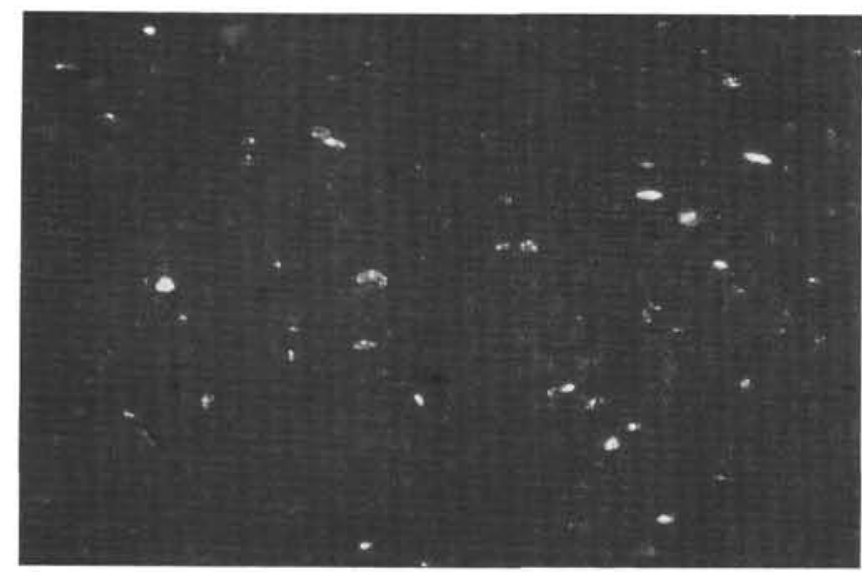

A

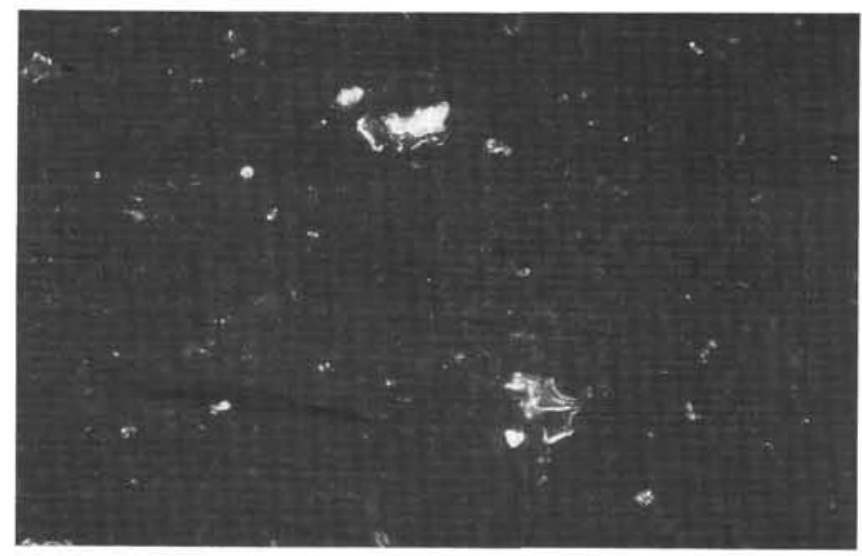

B

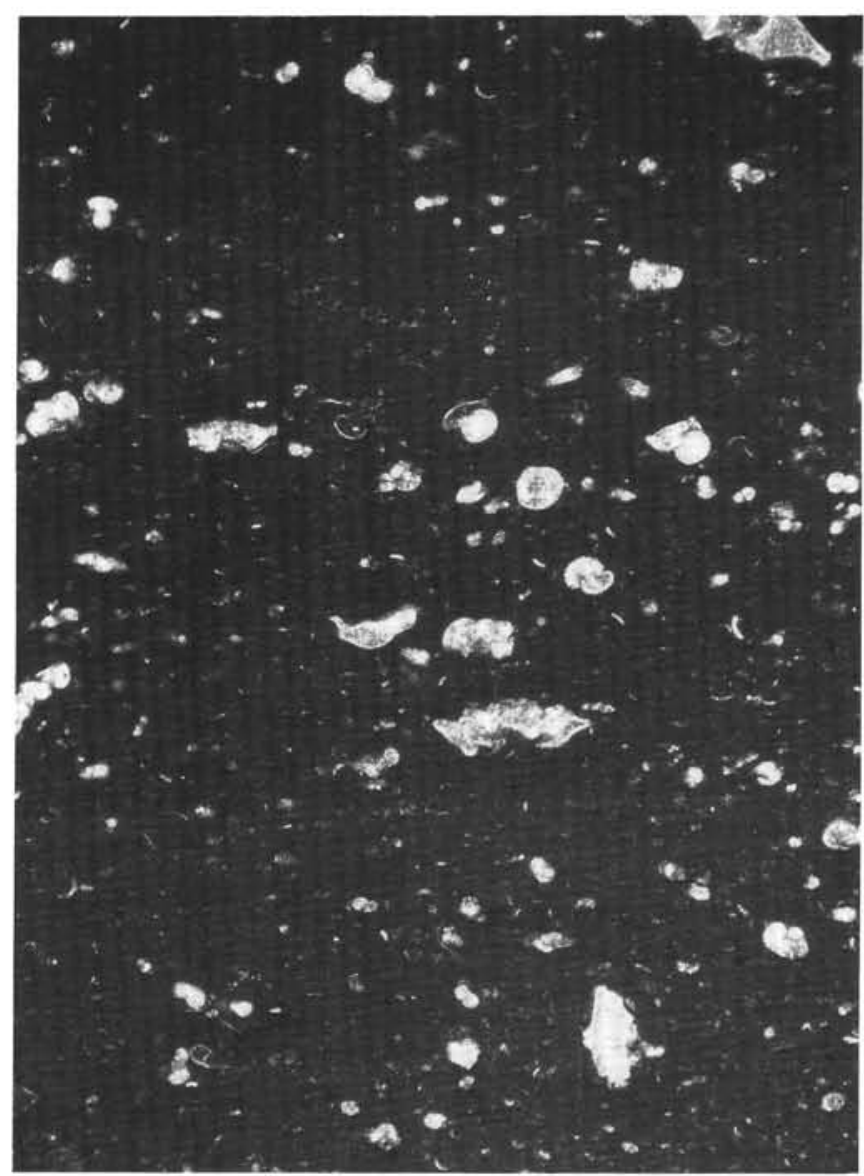

C

Figure 8. Photomicrographs of homogeneous microfacies from the Cretaceous sequence of Hole $807 \mathrm{C}$; all $30 \times(3 \mathrm{~cm}=1 \mathrm{~mm})$. A. Homogeneous microfacies with minute planktonic foraminifers, Sample 130-807C-67R-4, 102-104 cm. B. Homogeneous microfacies with background of minute planktonic foraminifers and rare ghosts of larger planktonic foraminifers; Sample 130-807C-63R-4, 57-59 cm. C. Homogeneous microfacies with broad size range of planktonic foraminifers: Sample 130-807C-59R-1, 77-78 cm.

carbonate on top of the plateau and the subsequent slumping along the margins as the plateau migrated northward into more carbonate productive waters. Significantly, the lower bathyal benthic assemblage is also found in these pulses, with only subtle changes in abundance and the addition of rare specimens and fragments of larger nodosariids. The degree of subtlety implies that the resedimented material also originated at bathyal water depths.

The abrupt appearance of laminated sediments in the upper Maastrichtian, on the other hand, likely results from passage of the site into a new current regime at a paleolatitude of $17^{\circ} \mathrm{S}$ (Gordon, 1990), and the subsequent increase in sediment redistribution. Although the trend toward smaller, globular planktonic foraminifers of the genera Globigerinelloides, Hedbergella, Heterohelix, and Pseudotextularia in the laminated interval could result from hydrologic sorting, we think the trend more likely resulted from the onset of increasingly eutrophic conditions, especially when combined with the recurrence of radiolarians.

\section{HIATUSES}

Hiatuses in the Cretaceous sequence from the Ontong Java Plateau are among the most extensive yet known from the vast Cretaceous Pacific Basin (Sliter, 1992; Kroenke et al., this volume). The importance of documenting the hiatuses is to determine those that are regional in extent and caused by local tectonic events or bottom currents vs. those that are widespread and caused by major changes in ocean circulation and chemistry. The hiatuses from the Ontong Java Plateau correlate with stratigraphic intervals in the Pacific that contain hiatuses recognized throughout the basin (Sliter, 1992). These include the late Aptian and Albian, Cenomanian to Turonian, Santonian to middle Campanian, and late Maastrichtian.

The intriguing aspect of the hiatuses from the Ontong Java Plateau is their stratigraphic extent, as shown in Figure 3. This has important bearing on the early history of the plateau in terms of the bathymetry and the paleoceanography of the mid-latitude waters traversed by means of plate motion. The best dated hiatuses occur within the carbonate sequence at Site 288 from the southern portion of the plateau (Fig. 1). A major hiatus over $15 \mathrm{~m} . \mathrm{y}$. long that includes most of the Albian occurs between Cores 30-288A-29 and -30 (Fig. 3 ). Following this hiatus, sedimentation continued without major breaks throughout the Cenomanian to the lower Santonian, based on the presence of current-winnowed and reworked planktonic foraminifers. The next major hiatus occurs within Core 30-288A-12 and includes much of the Santonian and Campanian (Andrews, Packham, et al., 1975). Finally, the uppermost Maastrichtian may be missing based on the calcareous nannofossils (Shafik, 1975), although the boundary falls in the uncored interval between Cores 30-288A-8 and -9 .

At Site 289, in contrast, a major hiatus extends from the late Aptian to the late Campanian (Fig. 3). The lowermost limestone in Cores 30- 


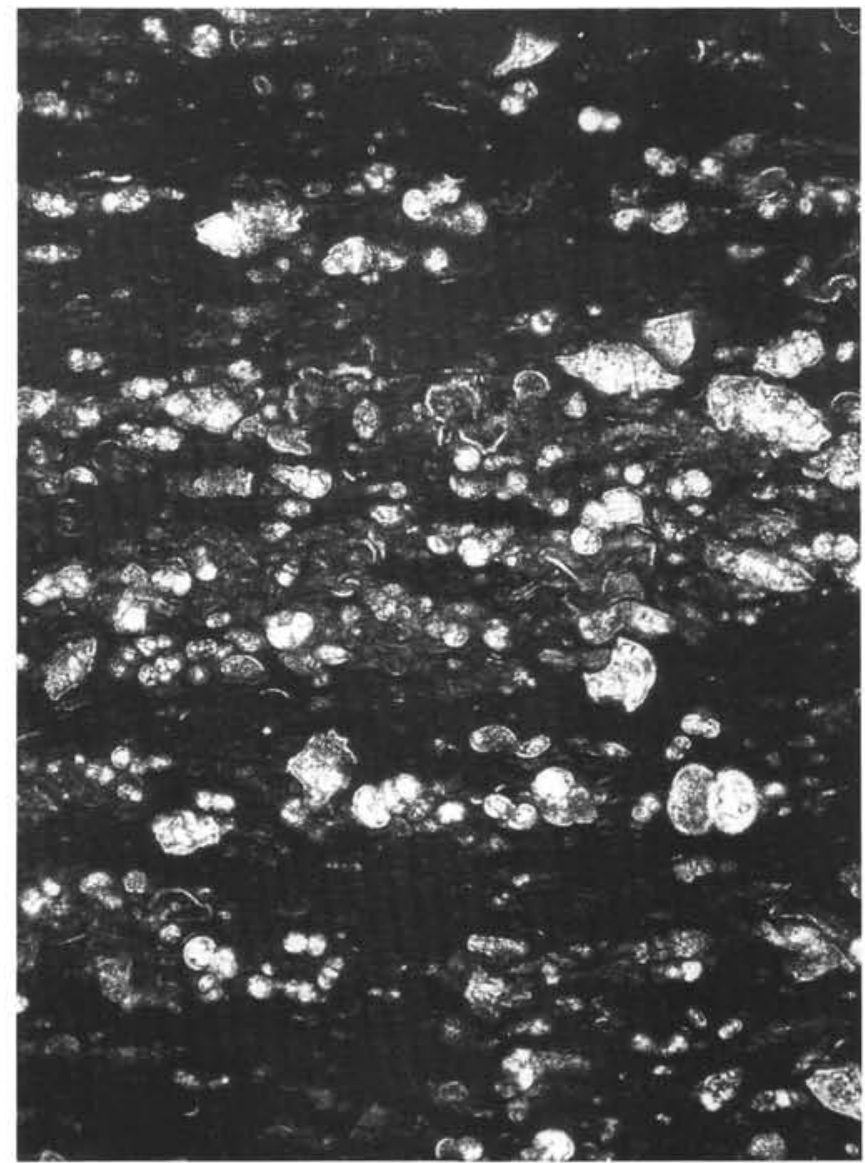

A

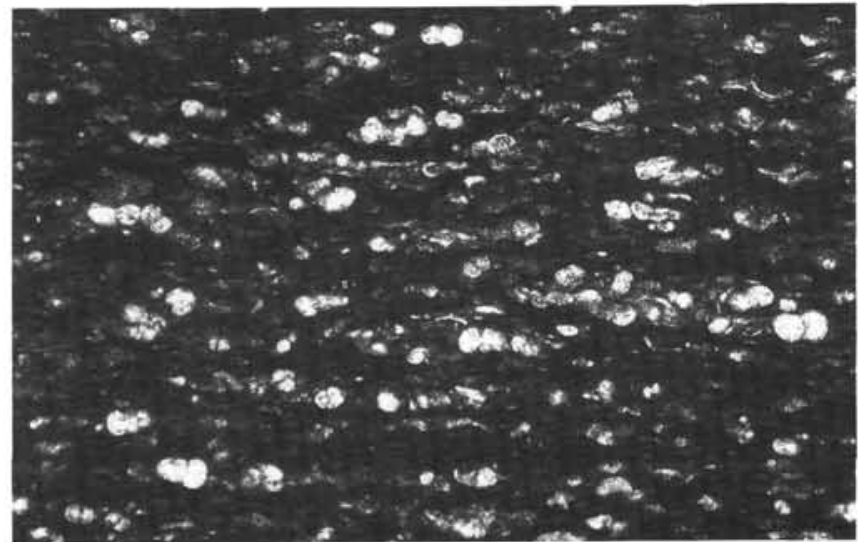

B

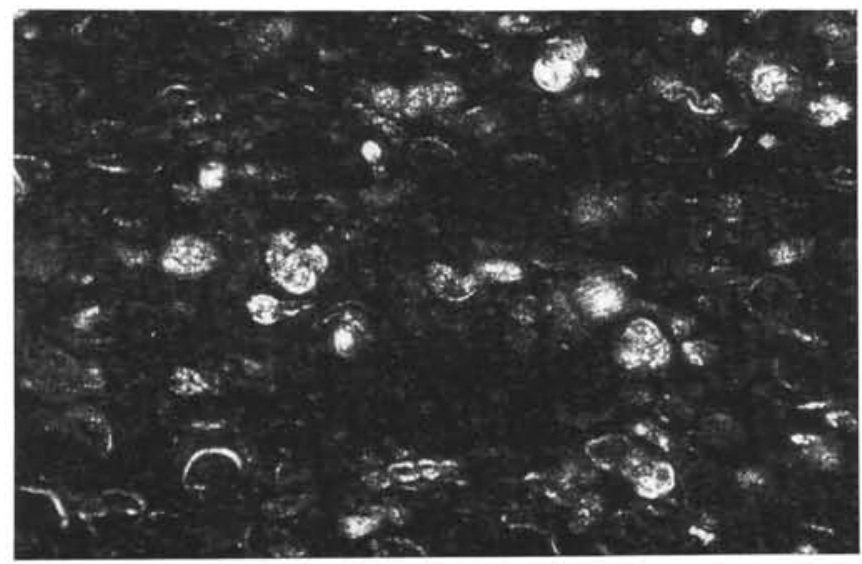

C

Figure 9. Photographs of laminated microfacies of Hole $807 \mathrm{C}$ showing sorting caused by resedimentation and pervasive dissolution; $(\mathbf{A}-\mathbf{B}) 30 \times(3 \mathrm{~cm}=1 \mathrm{~mm})$; (C) $60 \times(3 \mathrm{~cm}=500 \mu \mathrm{m})$. A. Layers of mostly medium- and small-sized planktonic foraminifers with globular chambers; Sample 130-807C-58R-1, 33-35 cm. B. Faint layers of small globular planktonic foraminifers with numerous ghosts and abundant fragments; Sample 130-807C-56R-3,13-15 cm. C. Dissolution facies in uppermost Maastrichtian near the Cretaceous/Tertiary boundary showing small globular planktonic foraminifers and abundant ghosts and fragments; Sample 130-807C-55R-1, 103-105 cm.

289-132 and $-131-2$ is middle to late Aptian in age whereas that of Core 289-131-1 is late Campanian. The mid Cretaceous, however, appears to be missing at Site 289 and may be condensed in the reddish brown tuff or, more likely, involved with an extended hiatus. The Upper Cretaceous sequence ranges from upper Campanian to middle Maastrichtian with an upper Maastrichtian hiatus found in Core 30289-122. The major difference between Sites 288 and 289 is the fivefold thicker Cretaceous sequence at Site 288. Although the midCretaceous interval at Site 288 is over $500 \mathrm{~m}$ below that of Site 289 in terms of depth below present sea level, the margin location of Site 288 protected the redeposited sediments, whereas those of Site 289 on top of the plateau were eroded or removed by bottom currents.

Thus, the extended hiatuses at Sites 803 and 807 are more similar to those of Site 289 than to those of Site 288 or to other sites in the Pacific Basin. Although the Cretaceous sequence at Site 807 is somewhat thicker than that at Site 289 , the greatly condensed sequence at Site 803 is ten times thinner. The recovery of late Albian radiolarians from Hole $803 \mathrm{D}$, therefore, provides the only biostratigraphic date from the Albian to Maastrichtian time interval at Sites 803 and 807 . We think that the lack of Cenomanian to Campanian sediments at these sites, which were paleobathymetrically deeper than Site 289, resulted from chemical rather than mechanical erosion. Regardless, erosion during this widespread hiatus took place at both intermediate and deeper water depths throughout the world ocean (Sliter, 1976).

\section{CONCLUSIONS}

The oldest dated sediments from the Ontong Java Plateau were recovered from Site 807 and are assigned to the Globigerinelloides blowi Zone of early Aptian age. Uncertainty in the exact meter level of the lowest limestone sample may indicate that oldest sediments overlying the basalt were not recovered. The Aptian age of the limestone from Site 807 is very similar to that of Site 289, but it is older than that of Site 288 where drilling ended before reaching basement.

The age of the oldest sediments at Site 803 is problematic. An Aptian to early Albian age is suggested by the foraminifers from the basal claystone, and radiolarians from overlying sediment yield a late Albian age. These ages conflict with a radiometric age of about $90 \mathrm{Ma}$ for the basaltic basement. The presence of interpillow limestone may suggest a hiatus at the basalt-sediment contact.

Deposition of the basal limestone at Sites 289,807 , and possibly 803 took place at lower bathyal depths below the foraminiferal lysocline but above the CCD. No evidence of reworked shallow-water biogenic material was found in the Leg 130 Cretaceous samples. Sediments at Site 807 record the transition to deposition beneath the $\mathrm{CCD}$ that is most likely related to thermal subsidence of the plateau and to a rise in the global CCD during the mid-Cretaceous.

Deposition at Site 807 continued beneath the CCD until the Maastrichtian or possibly the latest Campanian. At Site 803, deposition continued beneath the CCD until the Eocene although an appar- 


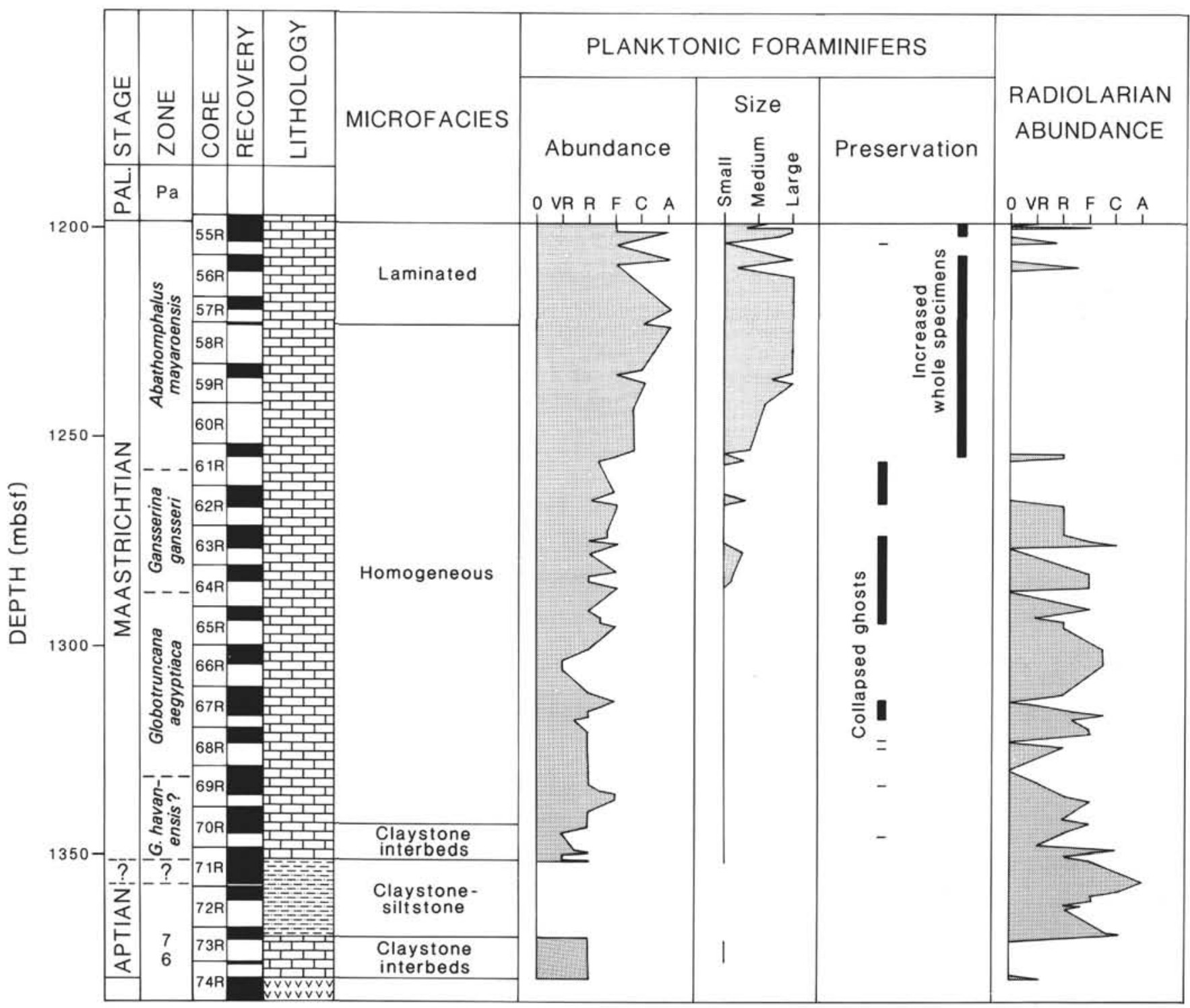

Figure 10. Relative abundance of planktonic foraminifers and radiolarians, and size distribution and preservation of larger planktonic foraminifers at Hole 807C. Abundance: $\mathrm{VR}=$ very rare, $\mathrm{R}=$ rare, $\mathrm{F}=$ few, $\mathrm{C}=$ common, and $\mathrm{A}=$ abundant. Other symbols as in Figure 5.

ently complete Cretaceous/Tertiary boundary is recorded in the claystone sequence by dissolution-resistant calcareous nannofossils. The presence of planktonic foraminifers from Site 288, however, demonstrates that sedimentation above the CCD took place on top of the plateau from the upper Albian to the lower Santonian.

Microfossils from Site 807 record a rapid drop in the CCD through the Maastrichtian and then a possible rise at the Cretaceous/Tertiary boundary. Throughout this interval, deposition took place at lower bathyal depths. Small planktonic foraminifers accumulated in redeposited fines derived from current winnowing atop the plateau whereas sorted, larger planktonic foraminifers were derived largely from repeated mass transport events. The increase in the pulses of larger planktonic foraminifers followed by the occurrence of laminated sediments, a marked increase in small, globular planktonic foraminifers, and an increase in radiolarians in the latest Maastrichtian suggests migration into more vigorous and productive subequatorial waters as a result of northward plate motion.

Hiatuses on Ontong Java Plateau, although extended by regional conditions, correlate with times of widespread hiatuses in the Pacific Basin. These include the late Aptian to late Albian, Cenomanian to
Turonian, Santonian to middle Campanian, and late Maastrichtian. The extended Aptian to Maastrichtian hiatus at Site 807 and the Cenomanian to Maastrichtian hiatus at Site 803 were the result of carbonate dissolution. The Santonian to late Campanian hiatus at Site 288 and the longer hiatus at Site 289 were caused by mechanical erosion on top of the plateau. The extent of the hiatuses attributed to mechanical erosion and the pervasive winnowing and reworking of foraminifers from Ontong Java Plateau compared with other Pacific sites may have resulted from the longitudinal position of the plateau relative to western boundary currents in the Cretaceous Pacific Ocean. In addition, mesoscale eddies and internal waves in the water column above the plateau likely contributed to the mechanical erosion during times of increased current flow.

\section{ACKNOWLEDGMENTS}

We are grateful to Mary McGann and Eleanor V. Kohnen for drafting the figures and assembling the tables. W.P. Elder, B.T. Huber, Kristin McDougall, and Isabella Premoli Silva reviewed this manuscript, and we appreciate their comments and suggestions. 


\section{REFERENCES*}

Andrews, J.E., Packham, G., et al., 1975. Init. Repts. DSDP, 30: Washington (U.S. Govt. Printing Office).

Arthur, M.A., Larson, R.L., and Dean, W.E., 1990. The Cretaceous mantlecrust-climate connection. Eos, 71:1660. (Abstract)

Berger, W.H., and Johnson, T.C., 1976. Deep-sea carbonates: dissolution and mass wasting on Ontong-Java Plateau. Science, 192:785-787.

Berger, W.H., Johnson, T.C., and Hamilton, E.L., 1977. Sedimentation on Ontong Java Plateau: observations on a classic "carbonate monitor." In Andersen, N.R., and Malahoff, A. (Eds.), The Fate of Fossil Fuel $\mathrm{CO}_{2}$ in the Oceans: New York (Plenum), 543-567.

Caldeira, K., and Rampino, M.R., 1990. Carbon dioxide emissions from Deccan volcanism and a K/T boundary greenhouse effect. Geophys. Res. Lett., 17:1299-1302.

, 1991. The mid-Cretaceous super plume, carbon dioxide, and global warming. Geophys. Res. Lett., 18:987-990.

Channell, J.E.T., Lowrie, W., and Medizza, F., 1979. Middle and early Cretaceous magnetic stratigraphy from the Cismon section, northern Italy. Earth Planet. Sci. Lett., 42:153-166.

Coccioni, R., Erba, E., and Premoli Silva, I., 1992. Barremian-Aptian calcareous plankton biostratigraphy from the Gorgo Cerbara section (Marche, central Italy) and implications for plankton evolution. Cretaceous Res.

Douglas, R.G., 1971. Cretaceous foraminifera from the northeastern Pacific Ocean: Leg 6, Deep Sea Drilling Project. In Fischer, A.G., Heezen, B.C., et al., Init. Repts. DSDP, 6: Washington (U.S. Govt. Printing Office), 1027-1053.

Duncan, R.A., and Clague, D.A., 1985. Pacific Plate motion recorded by linear volcanic chains. In Nairn, A.E.M., Stehli, F.G., and Uyeda, S. (Eds.), Ocean Basins and Margins (Vol. 7): The Pacific Basin: New York (Plenum), 89-121.

Gordon, R.G., 1990. Test for bias in paleomagnetically determined paleolatitudes from Pacific Plate Deep Sea Drilling Project sediments. J. Geophys. Res., 95:8397-8404.

Harland, W.B., Armstrong, R.L., Cox, A.V., Craig, L.E., Smith, A.G., and Smith, D.G., 1990. A Geologic Time Scale 1989: Cambridge (Cambridge Univ. Press).

Harland, W.B., Cox, A.V., Llewellyn, P.G., Pickton, C.A.G., Smith, A.G., and Walters, R., 1982. A Geologic Time Scale: New York (Cambridge Univ. Press).

Kent, D.V., and Gradstein, F.M., 1985. A Cretaceous and Jurassic geochronology. Geol. Soc. Am. Bull., 96:1419-1427.

Kroenke, L.W., Berger, W.H., Janecek, T.R., et al., 1991. Proc. ODP, Init. Repts., 130: College Station, TX (Ocean Drilling Program).

Larson, R.L., 1991a. Latest pulse of Earth: evidence for a mid-Cretaceous superplume. Geology, 19:547-550. 19:963-966. $1991 \mathrm{~b}$. Geological consequences of superplumes. Geology,

Leckie, R.M., and Bralower, T.J., 1991. New ages for planktonic foraminiferal datums within the Barremian-lower Aptian. Geol. Soc. Am., Abstr. Programs, 23:A167. (Abstract)

Richards, M.A., Jones, D.L., Duncan, R.A., and DePaolo, D.J., 1991. A mantle plume initiation model for the Wrangellia flood basalt and other oceanic plateaus. Science, 254:263-267.

Shafik, S., 1975. Nannofossil biostratigraphy of the southwest Pacific, DSDP Leg 30. In Andrews, J.E., Packham, G., et al., Init. Repts. DSDP, 30: Washington (U.S. Govt. Printing Office), 549-598.

Sigal, J., 1977. Essai de zonation du Crétacé méditerranéenne à l'aide des foraminifères planctoniques. Geol. Mediterr., 4:99-108.

Sliter, W.V., 1976. Cretaceous foraminifers from the southwestern Atlantic Ocean, Leg 36, Deep Sea Drilling Project. In Barker, P., Dalziel, I.W.D., et al., Init. Repts. DSDP, 36: Washington (U.S. Govt. Printing Office), 519-573.

, 1989. Biostratigraphic zonation for Cretaceous planktonic foraminifers examined in thin section. J. Foraminiferal Res., 19:1-19.

- 1992. Cretaceous planktonic foraminiferal biostratigraphy and paleoceanographic events in the Pacific Ocean with emphasis on indurated sediments. In Ishizaki, K., and Saito, T. (Eds.), Centenary of Japanese Micropaleontology: Tokyo (Terra Sci.), 281-299.

Tarduno, J.A., Sliter, W.V., Bralower, T.J., McWilliams, M., Premoli Silva, I., and Ogg, J.G., 1989. M-sequence reversals recorded in DSDP sediment

* Abbreviations for names of organizations and publication titles in ODP reference lists follow the style given in Chemical Abstracts Service Source Index (published by American Chemical Society). cores from the western Mid-Pacific Mountains and Magellan Rise. Geol. Soc. Am. Bull., 101:1306-1316.

Tarduno, J.A., Sliter, W.V., Kroenke, L., Leckie, M., Mayer, H., Mahoney, J.J., Musgrave, R., Storey, M., and Winterer, E.L., 1991. Rapid formation of Ontong Java Plateau by Aptian mantle plume volcanism. Science, 254:399-403.

Thierstein, H.R., 1973. Lower Cretaceous nannoplankton biostratigraphy. Abh. Geol. Bundesanst. (Austria) 29:3-52.

, 1979. Paleoceanographic implications of organic carbon and carbonate distribution in Mesozoic deep sea sediments. In Talwani, M., Hay, W., and Ryan, W.B.F. (Eds.), Deep Drilling Results in the Atlantic Ocean: Continental Margins and Paleoenvironment. Am. Geophys. Union, Maurice Ewing Ser., 3:249-274.

Tucholke, B.E., and Vogt, P.R., 1979. Western North Atlantic: sedimentary evolution and aspects of tectonic history. In Tucholke, B.E., Vogt, P.R., et al., Init. Repts. DSDP, 43: Washington (U.S. Govt. Printing Office), 791-825.

Vandenburg, J., Klootwijk, C.T., and Wonders, A.A.H., 1978. Late Mesozoic and Cenozoic movements of the Italian peninsula: further paleomagnetic data from the Umbrian sequence. Geol. Soc. Am. Bull., 89:133-150.

Whitechurch, H., Montigny, R., Sevigny, J., Storey, M., and Salters, V.J., 1992. $\mathrm{K}-\mathrm{Ar}$ and ${ }^{40} \mathrm{Ar}{ }^{39} \mathrm{Ar}$ ages of Central Kerguelen Plateau basalts. In Wise, S.W., Jr., Schlich, R., et al., Proc. ODP, Sci. Results, 120, Pt. 1: College Station, TX (Ocean Drilling Program), 71-77.

\section{Date of initial receipt: 27 January 1992 \\ Date of acceptance: 17 May 1992 \\ Ms 130B-017}

\section{APPENDIX}

\section{Faunal Reference List}

Abathomphalus mayaroensis $($ Bolli $)=$ Globotruncana mayaroensis Bolli Contusotruncana contusa $($ Cushman $)=$ Pulvinulina arca Cushman var. contusa Cushman

Contusotruncana fornicata $($ Cushman $)=$ Globotruncana fornicata Cushman

Contusotruncana patelliformis $($ Gandolfi $)=$ Globotruncana $($ Globotruncana $)$ contusa (Cushman) subsp. patelliformis Gandolfi

Contusotruncana plummerae $($ Gandolfi $)=$ Globotruncana $($ Globotruncana $)$ fornicata Plummer subsp. plummerae Gandolfi

Gansserina gansseri (Bolli) = Globotruncana gansseri Bolli

Globigerinelloides alvarezi (Eternod Olvera) = Planomalina alvarezi Eternod Olvera

Globigerinelloides blowi $($ Bolli $)=$ Planomalina blowi Bolli

Globigerinelloides duboisi $($ Chevalier $)=$ Globigerinella duboisi Chevalier

Globigerinelloides ferreolensis (Moullade) $=$ Biticinella ferreolensis Moullade

Globigerinelloides gottisi $($ Chevalier $)=$ Globigerinella gottisi Chevalier

Globigerinelloides maridalensis $($ Bolli) $=$ Planomalina maridalensis Bolli

Globigerinelloides messinae $($ Brönnimann $)=$ Globigerinella messinae mess inae Brönnimann

Globigerinelloides prairiehillensis Pessagno

Globigerinelloides subcarinatus (Brönnimann) = Globigerinella messinae subcarinata Brönnimann

Globotruncana aegyptiaca Nakkady

Globotruncana arca $($ Cushman $)=$ Pulvinulina arca Cushman

Globotruncana bulloides Vogler

Globotruncana hilli Pessagno

Globotruncana linneiana (d'Orbigny) = Rosalina linneiana d'Orbigny

Globotruncana mariei Banner and Blow

Globotruncana ventricosa White

Globotruncanella havanensis $($ Voorwijk $)=$ Globotruncana havanensis Voorwijk

Globotruncanella petaloidea $($ Gandolfi $)=$ Globotruncana $($ Rugoglobigerina $)$ petaloidea Gandolfi subsp. petaloidea Gandolfi

Globotruncanita stuarti (de Lapparent) = Rosalina stuarti de Lapparent

Globotruncanita stuartiformis (Dalbiez) = Globotruncana (Globotruncana) elevata Brotzen subsp. stuartiformis Dalbiez

Gublerina robusta de Klasz

Guembelitria cretacea Cushman

Hedbergella delrioensis (Carsey) = Globigerina cretacea d'Orbigny var. delrioensis Carsey

Hedbergella hispaniae Longoria

Hedbergella holmdelensis Olsson 
Hedbergella monmouthensis $($ Olsson $)=$ Globorotalia monmouthensis Olsson Hedbergella occulta Longoria

Hedbergella praetrocoidea Kretzschmar and Gorbatchik

Hedbergella sigali Moullade

Hedbergella similis Longoria

Hedbergella trocoidea $($ Gandolfi) $=$ Anomalina lorneiana $(\mathrm{d}$ 'Orbigny) var trocoidea Gandolfi

Heterohelix glabrans $($ Cushman $)=$ Guembelina glabrans Cushman

Heterohelix globulosa $($ Ehrenberg) $=$ Textularia globulos $a$ Ehrenberg

Heterohelix pulchra $($ Brotzen $)=$ Guembelina pulchra Brotzen

Heterohelix striata $($ Ehrenberg $)=$ Textularia striata Ehrenberg
Leupoldina reicheli $($ Bolli $)=S$ chackoina reicheli Bolli

Planoglobulina acervulinoides $($ Egger $)=$ Guembelina acervulinoides Egger

Pseudoguembelina costulata $($ Cushman $)=$ Guembelina costulata Cushman

Pseudoguembelina excolat $a($ Cushman $)=$ Guembelina excolata Cushman

Pseudotextularia elegans $($ Rzehak $)=$ Cuneolina elegans Rzehak

Rugoglobigerina hexacamerata Brönnimann

Rugoglobigerina rugosa $($ Plummer $)=$ Globigerina rugosa Plummer

Shackoina multispinata $($ Wickenden $)=$ Hantkenina multispinata Cushman and Wickenden

Ticinella bejaouaensis Sigal

Trinitella scotti Brönnimann 


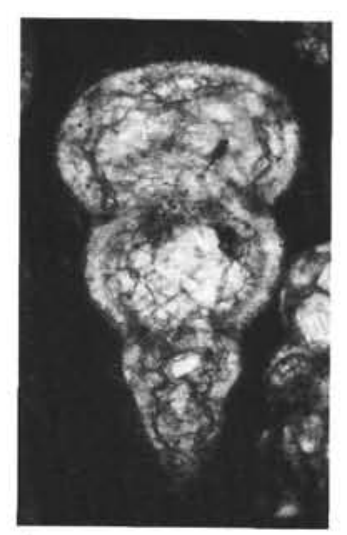

1

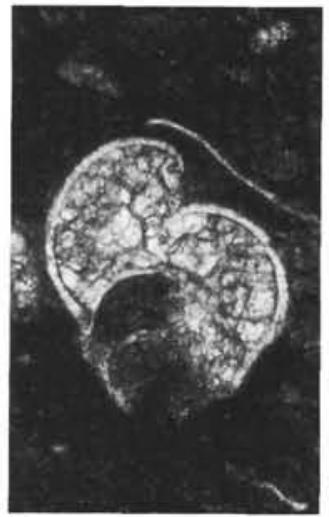

2

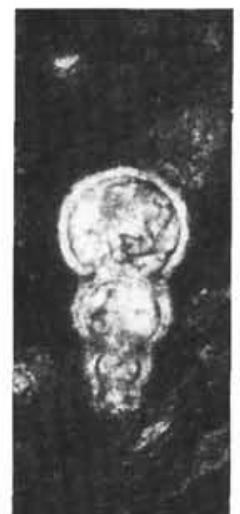

3

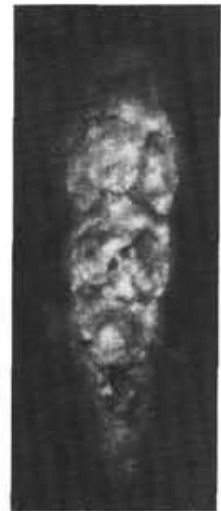

4

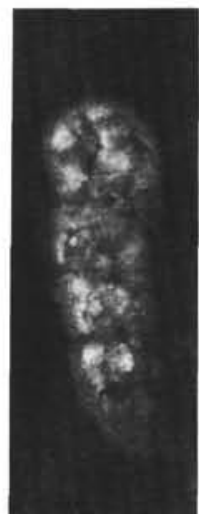

5

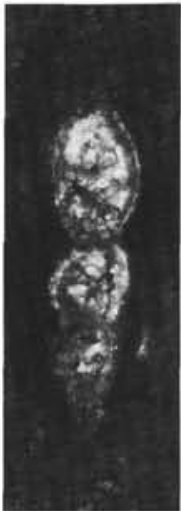

6

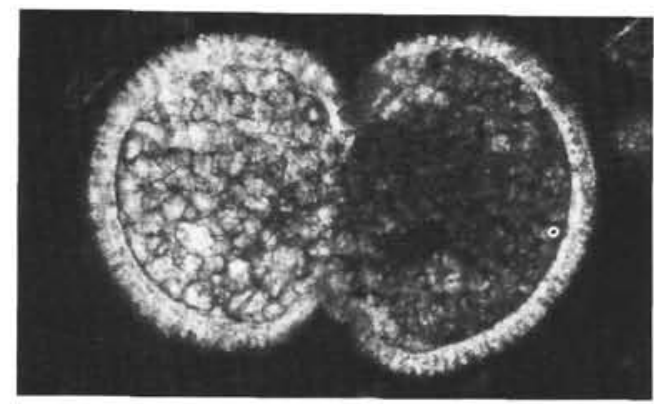

7

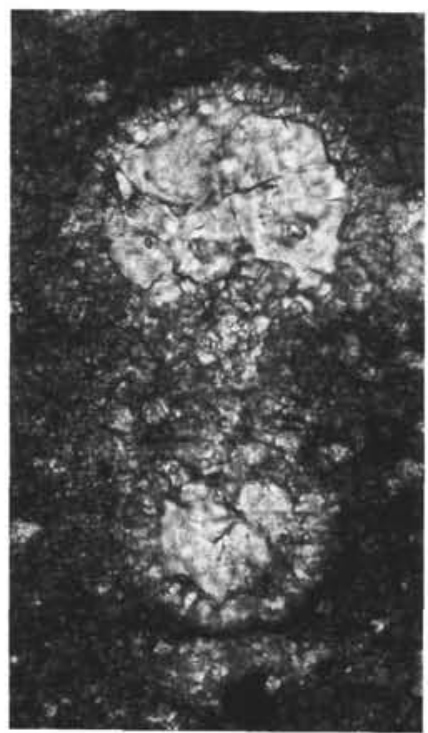

10

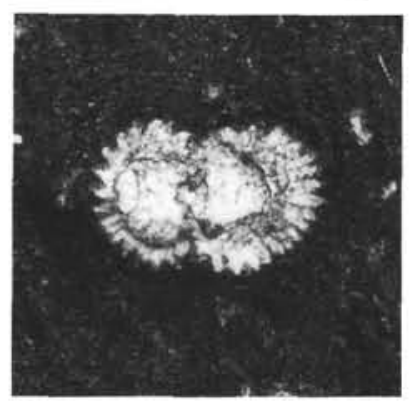

8

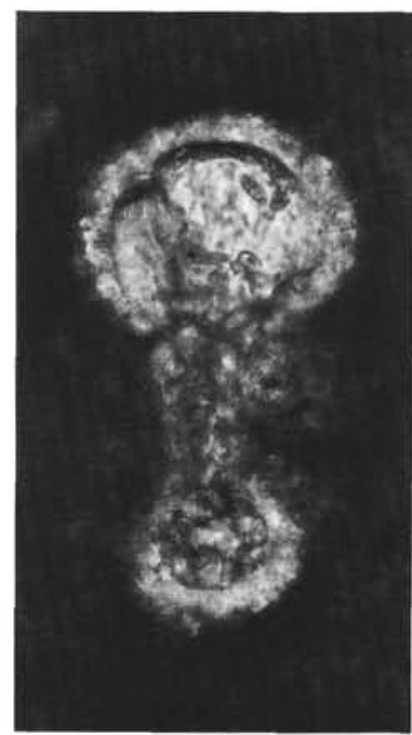

11

12

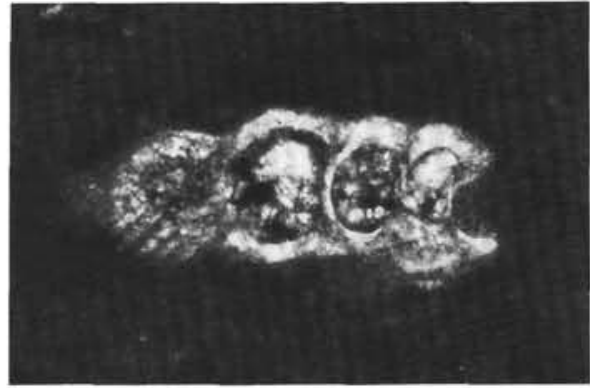

9

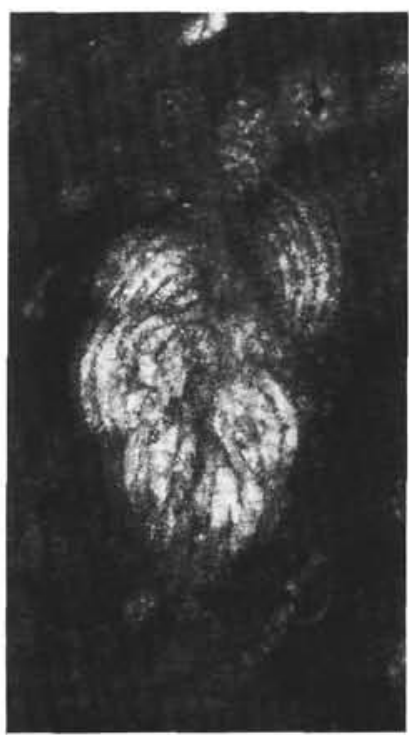

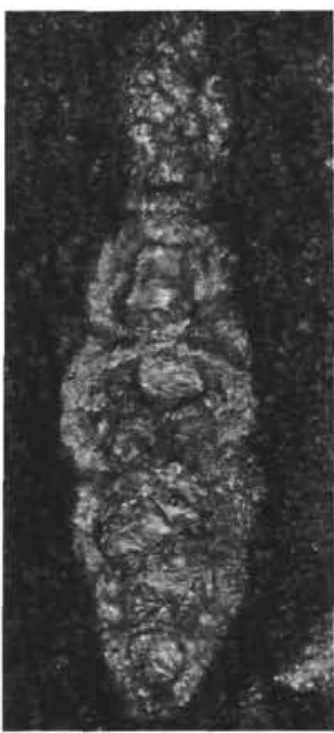

13

Plate 1. Thin section photomicrographs of Cretaceous planktonic foraminifers from ODP Hole 807C and DSDP Site 289. Figures 1-2, 5-9, and 12 are 150x $(3 \mathrm{~cm}=200 \mu \mathrm{m})$; Figures $3-4,10-11$, and 13 are $300 \times(3 \mathrm{~cm}=100 \mu \mathrm{m})$. 1, 7. Pseudotextularia elegans (Rzehak); (1) edge section, Sample 130-807C-59R-CC; (7) transverse section, Sample 130-807C-59R-2,99-100 cm. 2. Heterohelix pulchra (Brotzen), lateral section, Sample 130-807C-59R1, 77-78 cm. 3. Heterohelix striata (Ehrenberg), edge section, Sample 130-807C-59R-CC. 4-5. Heterohelix globulosa (Ehrenberg); (4) edge section, Sample 130-807C-64R-2, 7-9 cm; (5) edge section, Sample 130-807C-64R-1, 64-66 cm. 6. Heterohelix glabrans (Cushman), edge section, Sample 130-807C-59R-CC. 8, 12. Pseudoguembelina costulata (Cushman); (8) transverse section showing prominent costae, Sample 130-807C-59R-1, 77-78 cm; (12) view of lateral surface, Sample 130-807C-59R-CC. 9, 13. Planoglobulina acervulinoides (Egger); (9) oblique transverse section of late stage with chamber proliferation in single plane, Sample 130-807C-61R-1, 50-52 cm; (13) edge section showing thickened wall of early biserial stage and later planar stage, Sample 130-807C-59R-1, 77-78 cm. 10. Globigerinelloides ferreolensis (Moullade), axial section, Sample 30-289-132-2, 37-40 cm. 11. Globigerinelloides prairiehillensis Pessagno, axial section, Sample 130-807C-61R-2, 50-52 cm. 


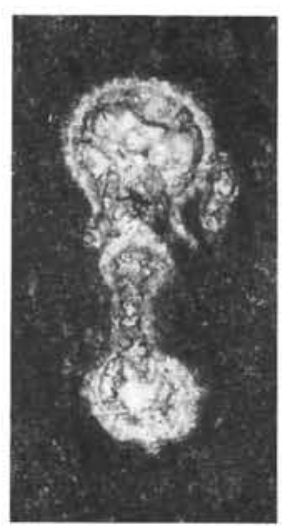

1

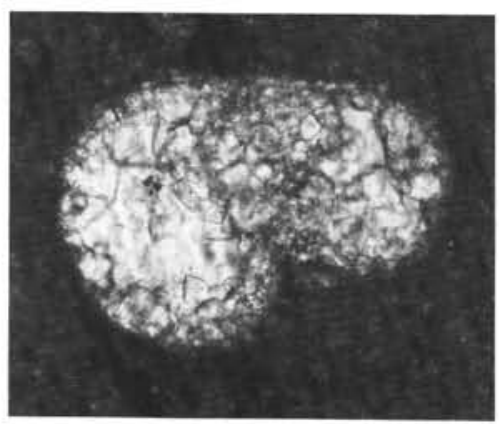

7

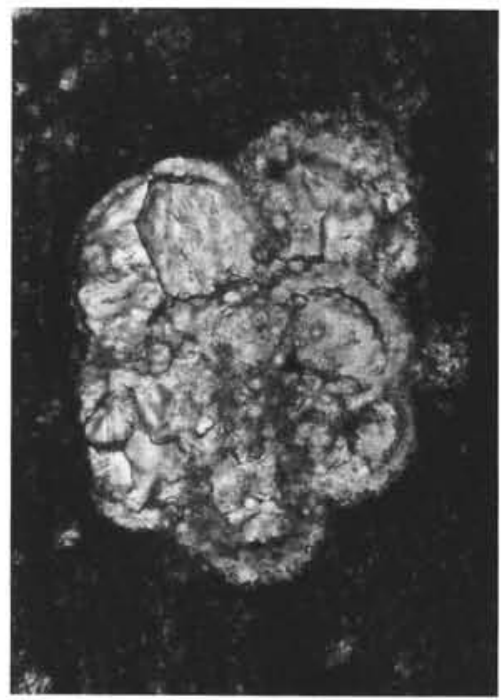

10

2

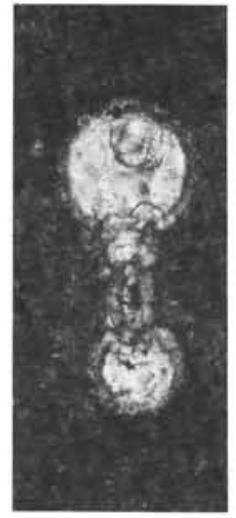

3

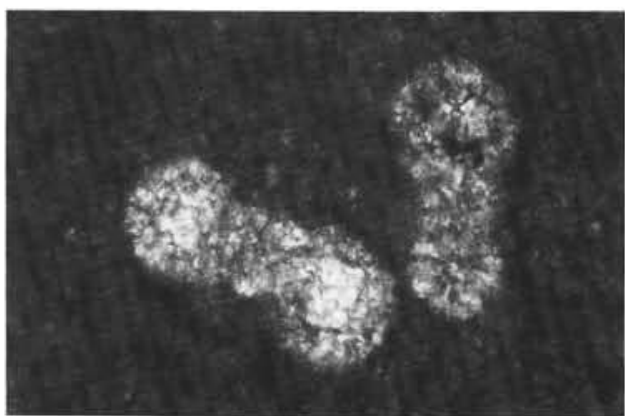

8

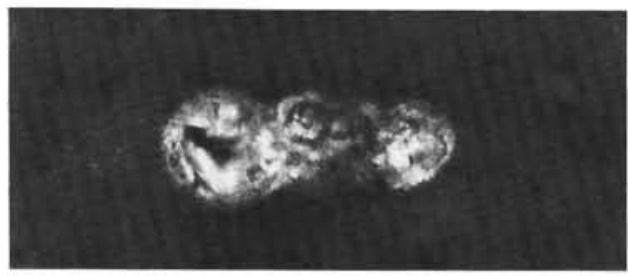

11

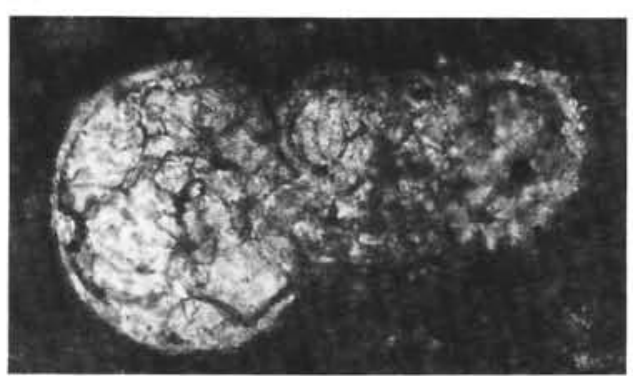

13

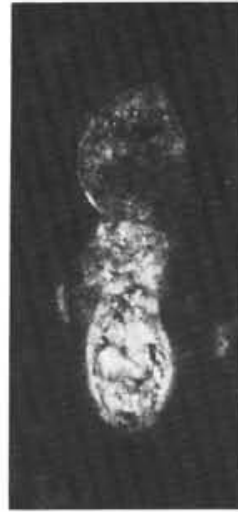

5

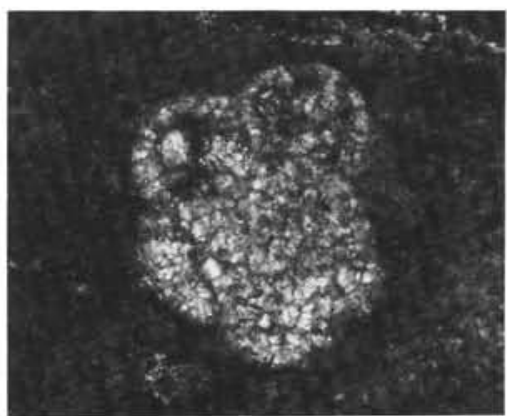

9

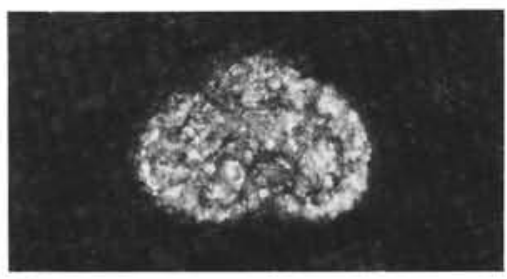

12

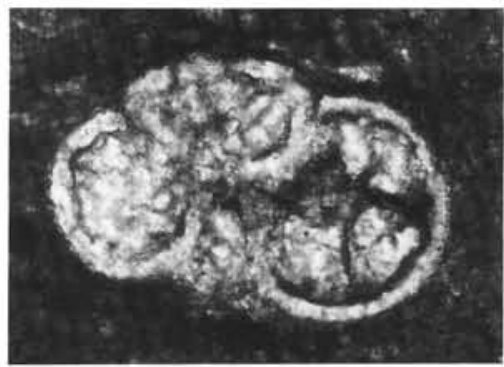

14

Plate 2. Thin section photomicrographs of Cretaceous planktonic foraminifers from ODP Hole $807 \mathrm{C}$ and DSDP Site 289. Figures 1 and $4-5$ are $150 \times(3 \mathrm{~cm}=$ $200 \mu \mathrm{m})$; Figures $2-3$ and 6-14 are $300 \times(3 \mathrm{~cm}=100 \mu \mathrm{m})$. 1-2. Globigerinelloides alvarezi (Eternod Olvera), axial sections; (1) Sample 130-807C-59R-1, $77-78 \mathrm{~cm}$; (2) small specimen, Sample 130-807C-67R-2, 46-48 cm. 3. Globigerinelloides blowi (Bolli), axial section showing aperture, Sample 130-807C-73R2, 115-117 cm. 4-5. Globigerinelloides messinae (Brönnimann), axial sections, Sample 130-807C-60R-CC. 6. Globigerinelloides subcarinatus (Brönnimann), axial section, Sample 130-807C-60R-CC. 7. Hedbergella hispaniae Longoria, axial section, Sample 130-807C-73R-CC. 8. Hedbergella similis Longoria on left, Globigerinelloides gottisi (Chevalier) on right, axial sections, Sample 130-807C-74R-1, 23-25 cm. 9. Hedbergella sp. cf. H. occulta Longoria, transverse section, Sample 130-807C-74R-1, 23-25 cm. 10. Hedbergella praetrocoidea Kretzschmar and Gorbatchik, transverse section, Sample 30-289-132-2, $37-40 \mathrm{~cm}$. 11. Hedbergella holmdelensis Olsson, axial section, Sample 130-807C-65R-CC. 12. Hedbergella sigali Moullade, axial section, Sample 130-807C$74 \mathrm{R}-1,23-25 \mathrm{~cm}$. 13. Hedbergella monmouthensis (Olsson), axial section, Sample 130-807C-59R-CC. 14. Hedbergella delrioensis (Carsey), axial section, Sample 30-289-132-2, 37-40 cm. 


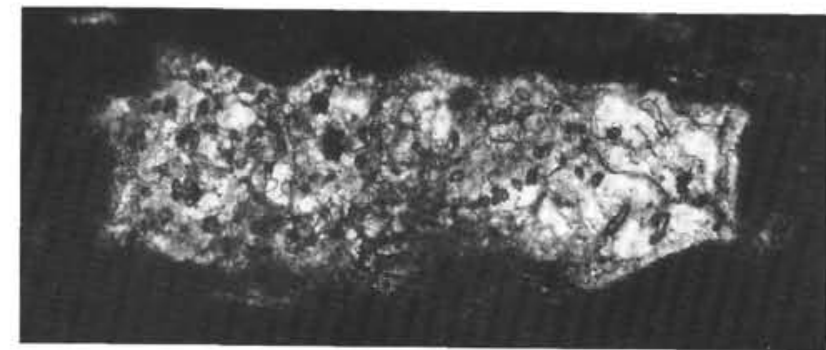

1

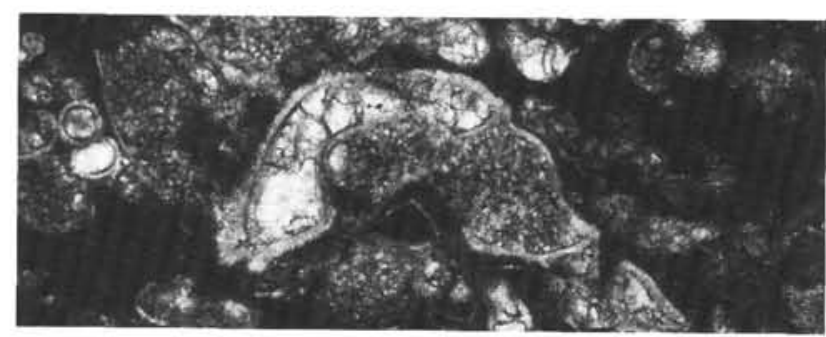

3

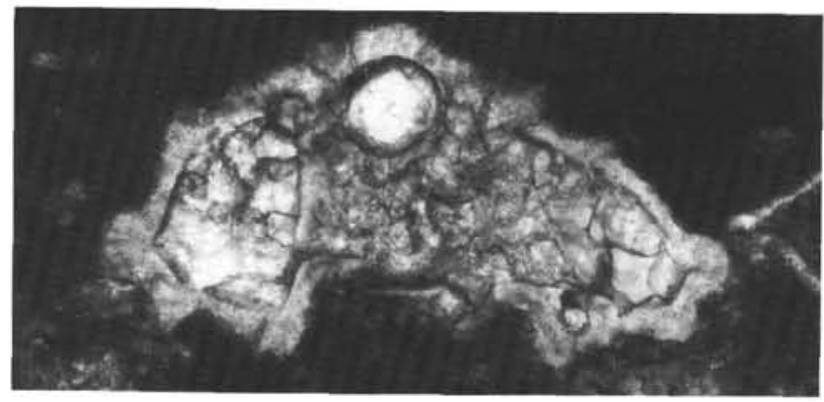

4

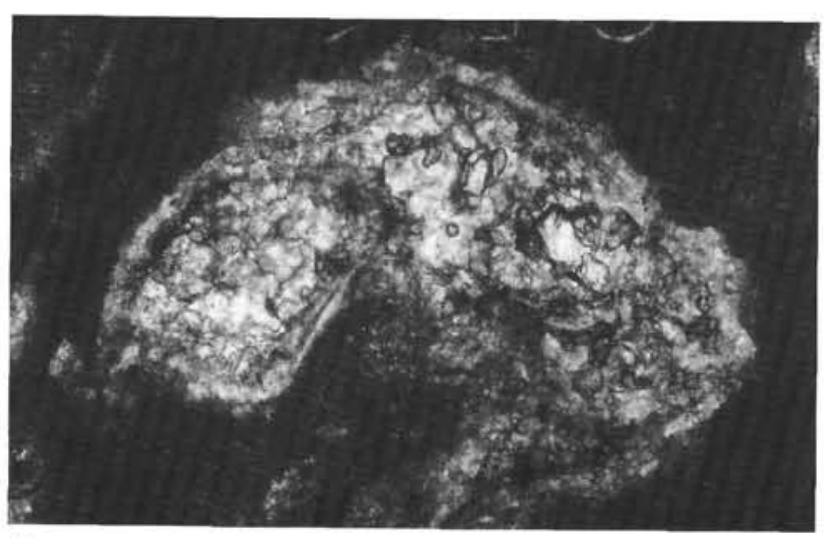

5

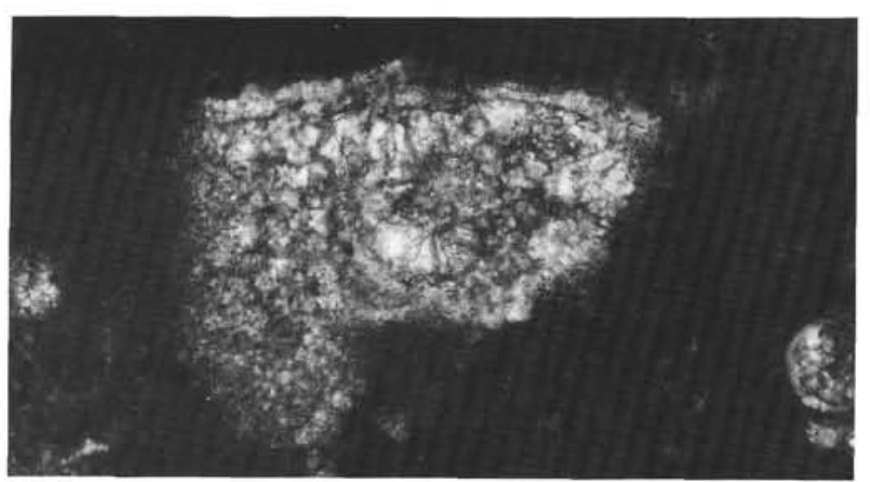

2

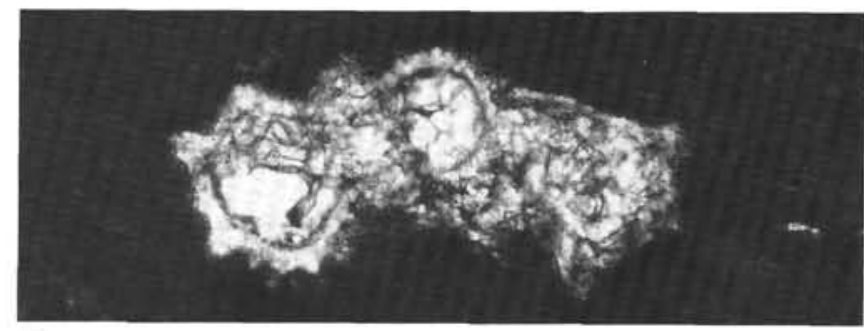

6

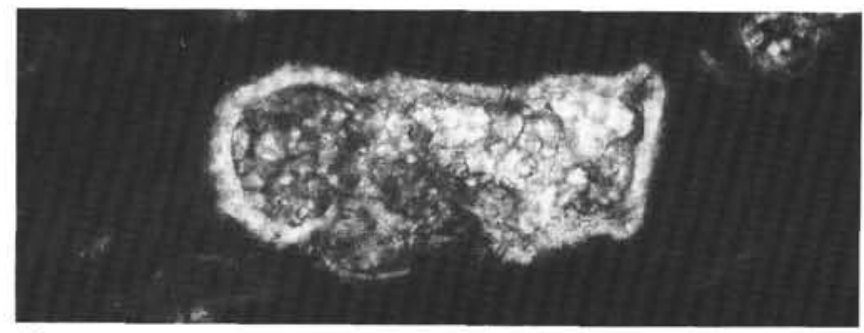

7

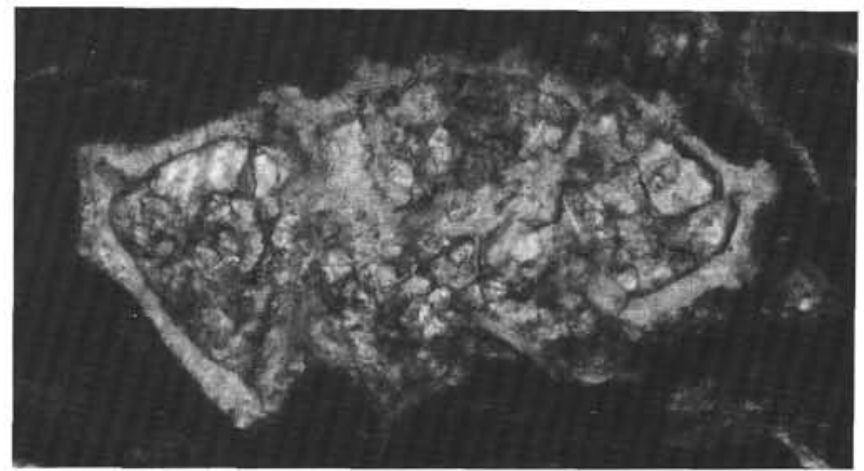

8

Plate 3. Thin section photomicrographs of Cretaceous planktonic foraminifers from ODP Hole $807 \mathrm{C}$. All axial sections. All except Figure $3,150 \times(3 \mathrm{~cm}=$ $200 \mu \mathrm{m})$; Figure $3,60 \times(3 \mathrm{~cm}=500 \mu \mathrm{m})$. 1. Globotruncana linneiana (d'Orbigny), Sample 130-807C-59R-CC. 2. Gansserina gansseri (Bolli), Sample 130-807C-59R-CC. 3. Contusotruncana contusa (Cushman), Sample 130-807C-57R-CC. 4. Contusotruncana fornicata (Cushman), Sample 130-807C57R-CC. 5. Contusotruncana patelliformis (Gandolfi), Sample 130-807C-59R-CC. 6. Globotruncana bulloides Vogler, Sample 130-807C-60R-1, 4-5 cm. 7. Globotruncana hilli Pessagno, Sample 130-807C-58R-1,33-35 cm. 8. Globotruncana aegyptiaca Nakkady, Sample 130-807C-58R-1, 33-35 cm. 


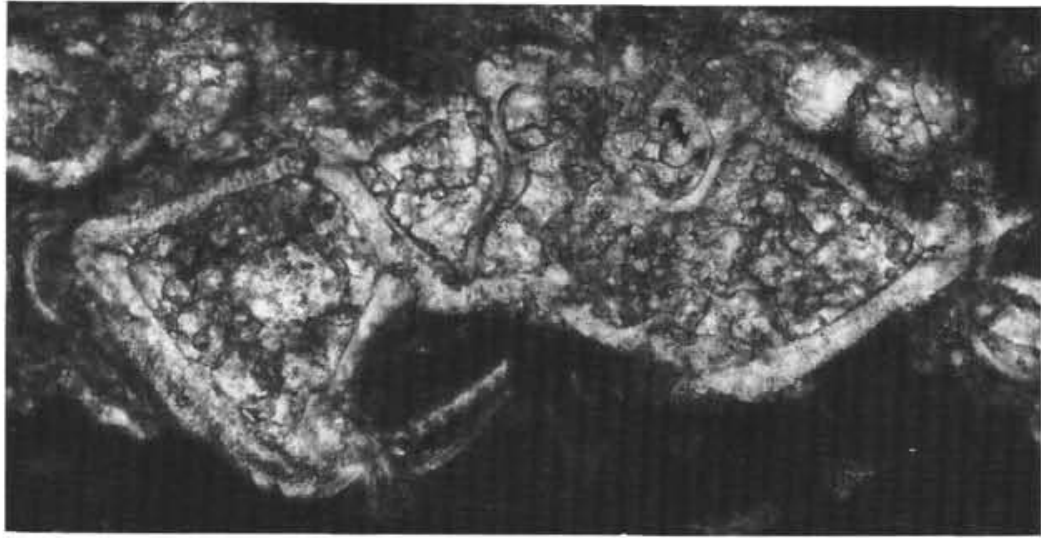

1

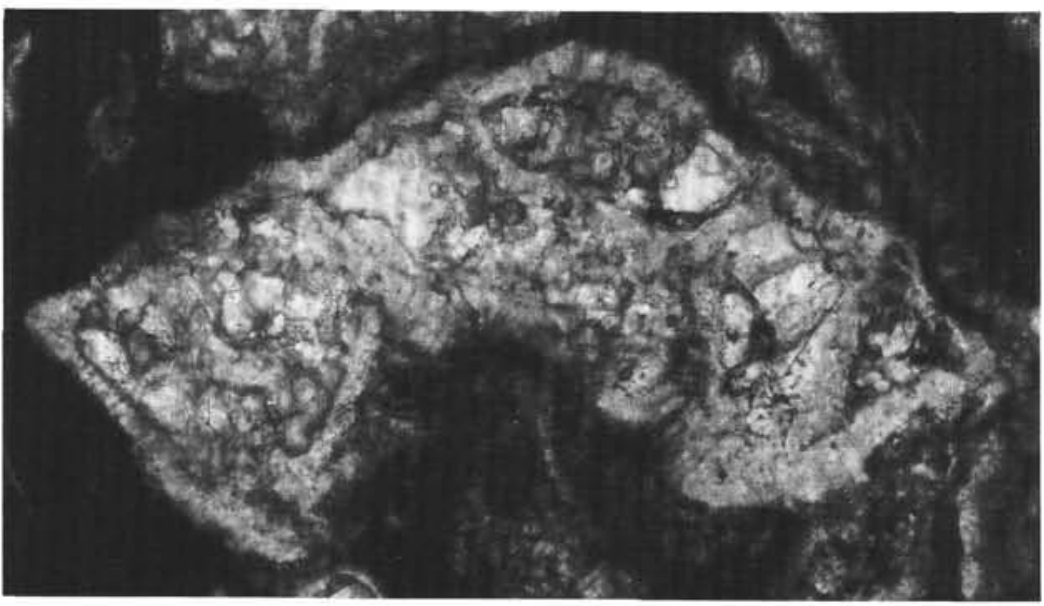

2

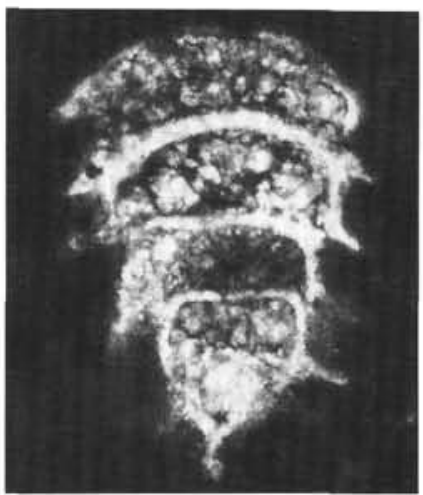

6

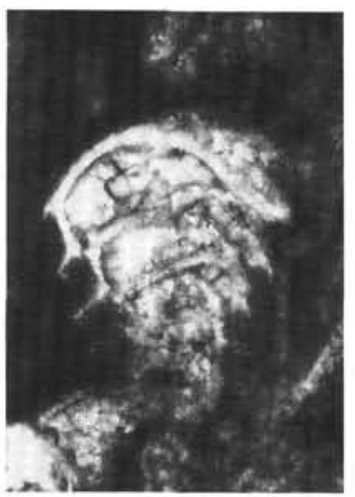

7

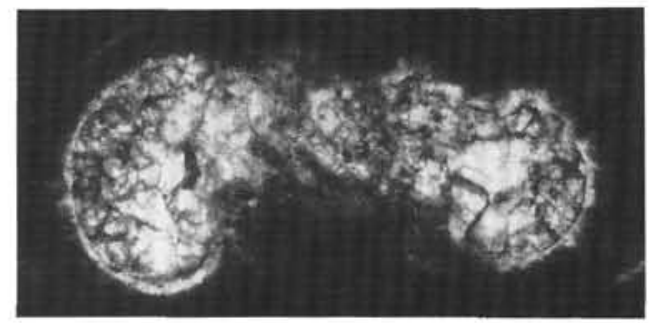

3

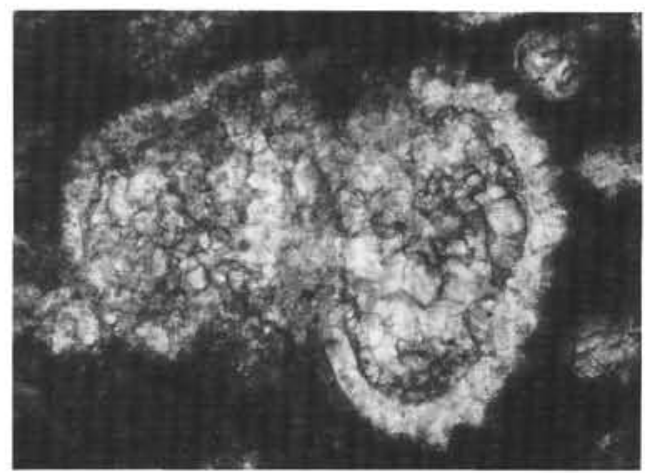

4

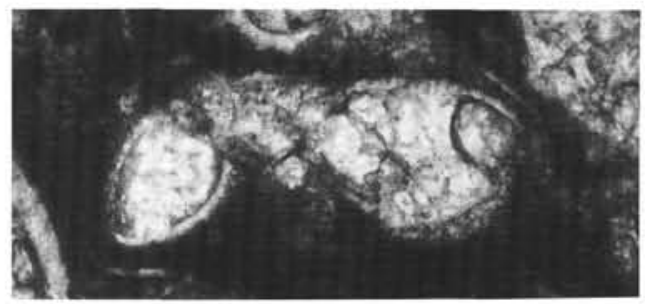

5

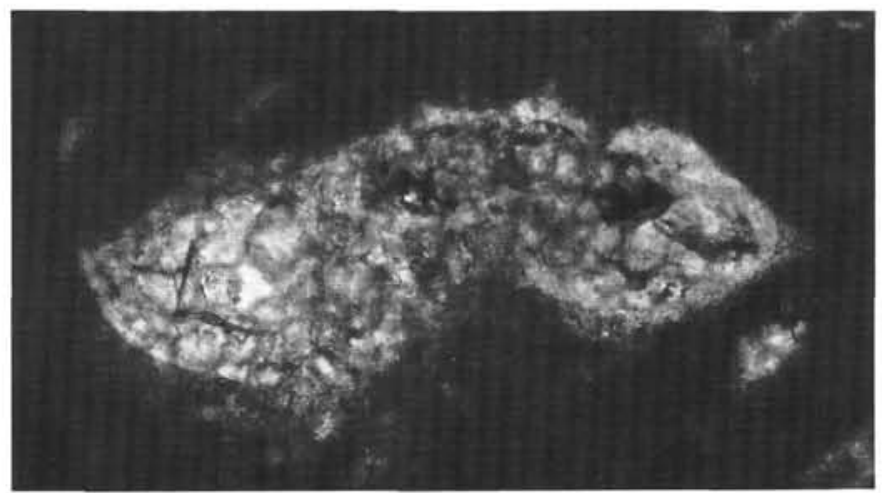

8

Plate 4. Thin section photomicrographs of Cretaceous planktonic and benthic foraminifers from ODP Hole $807 \mathrm{C}$. All except Figure $8,150 \times(3 \mathrm{~cm}=200 \mu \mathrm{m})$; Figure 8, 300 $(3 \mathrm{~cm}=100 \mu \mathrm{m})$. 1. Globotruncanita stuartiformis (Dalbiez), axial section, Sample 130-807C-58R-1, 33-35 cm. 2. Globotruncanita stuarti (de Lapparent), axial section, Sample 130-807C-58R-1, 33-35 cm. 3. Rugoglobigerina hexacamerata Brönnimann, axial section, Sample 130-807C-60R-CC. 4. Rugoglobigerina rugosa (Plummer), axial section, Sample 130-807C-55R-2, 93-95 cm. 5. Globotruncanella petaloidea (Gandolfi), axial section, Sample 130-807C-57R-CC. 6-7. Aragonia sp.; (6) edge section illustrating characteristic sutural extensions, Sample 130-807C-61R-CC; (7) lateral section, Sample 130-807C-57R-CC. 8. Globotruncanella havanensis (Voorwijk), axial section, Sample 130-807C-60R-CC. 


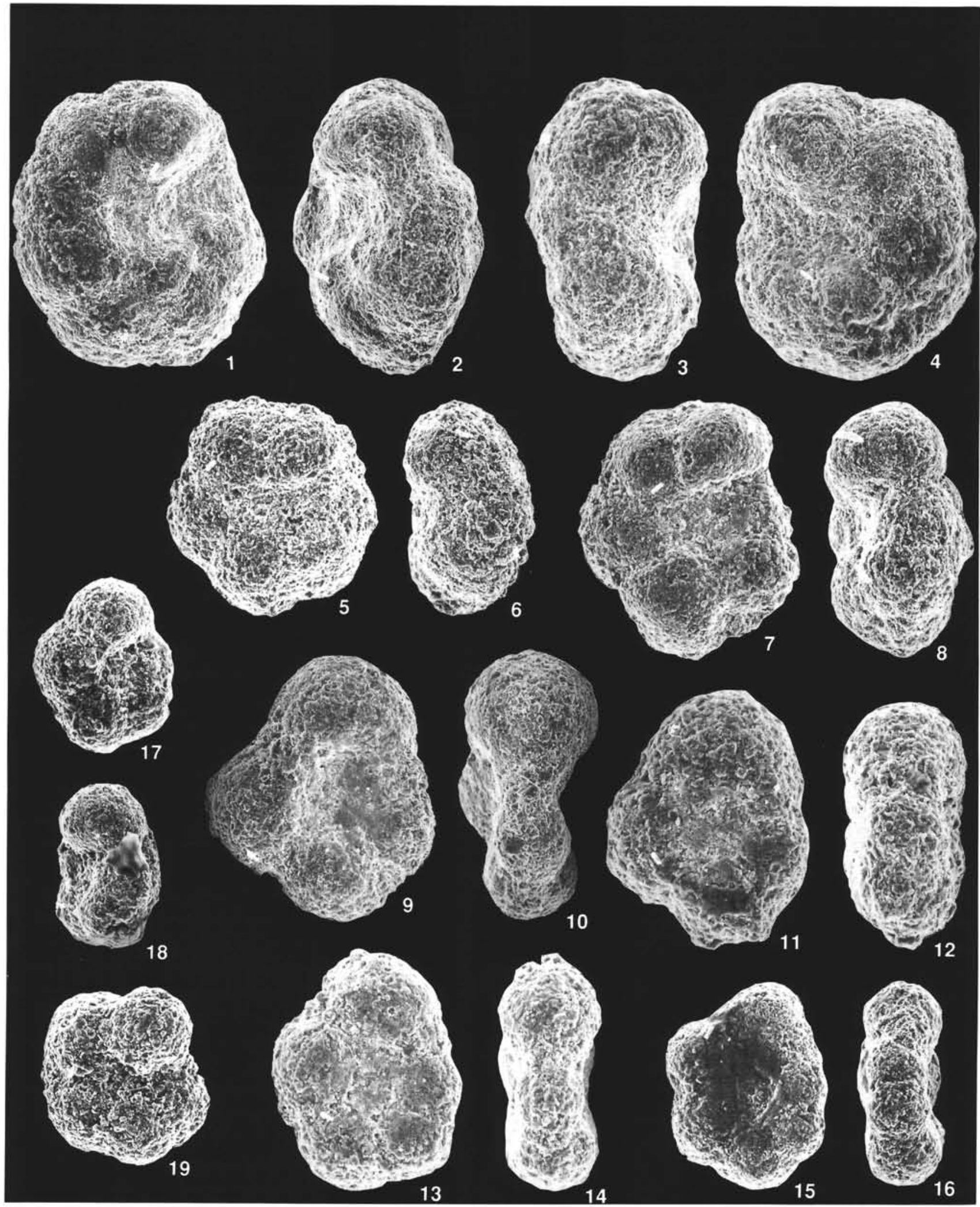

Plate 5. 1-2. Ticinella (?) bejaouaensis Sigal, umbilical and side views, Sample 30-289-131-3, 80-82 cm, 150x. 3-4. Hedbergella trocoidea (Gandolfi), side and umbilical views, Sample 30-289-131-3,80-82 cm, 200×. 5-8. Hedbergella praetrocoidea Kretzschmar and Gorbatchik, umbilical and side views, Sample 30-289-132-1, 21-23 cm, 200x. 9-12. Globigerinelloides blowi (Bolli), side and edge views, Sample 30-289-132-1, 21-23 cm; (9-10) 200x; (11-12) 300x. 13-14. Globigerinelloides gottisi (Chevalier), side and edge views, Sample 30-289-132-1,21-23 cm, 300×. 15-16. Globigerinelloides sp. cf. G. gottisi (Chevalier), side and edge views, Sample 30-289-132-1, 21-23 cm, 200×. 17-18. Hedbergella sp. cf. H. infracretacea (Glaessner), umbilical and side views, Sample 30-289-132-1, 21-23 cm, 200x. 19. Hedbergella sp. cf. H. excelsa Longoria, umbilical view, Sample 30-289-132-1, 21-23 cm, 200x. 